$\mathrm{DFPD} 10 / \mathrm{TH} / 03$

February, 2010

\title{
Pure Spinor Approach to Type IIA Superstring Sigma Models and Free Differential Algebras
}

\author{
Mario Tonin ${ }^{1}$ \\ Dipartimento di Fisica, Universita degli Studi di Padova, \\ INFN, Sezione di Padova, \\ Via F. Marzolo 8, 35131 Padova, Italy
}

\begin{abstract}
This paper considers the Free Differential Algebra and rheonomic parametrization of type IIA Supergravity, extended to include the BRS differential and the ghosts. We consider not only the ghosts $\lambda$ 's of supersymmetry but also the ghosts corresponding to gauge and Lorentz transformations. In this way we can derive not only the BRS transformations of fields and ghosts but also the standard pure spinor constraints on $\lambda$ 's. Moreover the formalism allows to derive the action for the pure spinor formulation of type IIA superstrings in a general background, recovering the action first obtained by Berkovits and Howe.
\end{abstract}

\footnotetext{
${ }^{1}$ E-mail address: mario.tonin@pd.infn.it
} 


\section{Introduction}

The pure spinor formulation of superstrings [1] is a very powerful method to provide a covariant quantization of superstring theories in flat superspace [2] - [5] or in special backgrounds like for instance $A d S_{5} \times S_{5}[6]$, but it is also important to describe superstrings in general backgrounds [7]- [12], especially in presence of RR fluxes.

The pure spinor formulation of $\sigma$-models to lowest order in $\alpha^{\prime}$ has been developed some time ago in [7], both for heterotic and type II superstrings (for heterotic strings see also [8]; for first order $\alpha^{\prime}$ corrections in the heterotic case, at the cohomological level, see [9].) The method of [7] starts by writing the more general action invariant under worldsheet conformal transformations and then derives the constraints of the background superfields by requiring nilpotence of the BRST charge and holomorphicity of the BRST currents (or equivalently invariance of the action under the BRST charge [10]).

Recently D'Auria, Grassi, Fre' and Trigiante [12], working in the case of type IIA ${ }^{2}$ superstring, have proposed an alternative method that reverses this procedure: they start from the geometrical formulation of type IIA supergravity and by generalizing a procedure [18], [19] well known for Yang-Mills theories, to which we shall refer as the method of the Extended (Free) Differential Algebra, they derive the constraints for the ghosts and the BRST invariant $\sigma$-model action. However the constraints that they obtain are not standard. Moreover these constraints, even if imply for the ghosts the same number of d.o.f.'s of the standard constraints, as they argue, are quite involved and lead to a complicated action.

In this paper we present a variant of the approach of [12] for type IIA $\sigma$-models that instead leads to the standard constraints on pure spinors and to the pure spinor action of [7]. The type IIB case presumably can be treated in a similar way and hopefully will be presented elsewhere.

The paper is organized as follows. Section II deals with the geometrical description of type IIA supergravity. In particular, after fixing notation, we present the supergravity constraints to be used in the next sections. For that, we adopt the parametrization of the curvatures derived in [12], [16]. In Section III we explain the approach of the Extended Differential Algebra and we derive the BRST algebra and the standard constraints on pure spinors. In Section IV we obtain the pure spinor action. The derivation of a property needed to assure the consistency of this action is given in the Appendix.

\section{Review of the geometrical description of $\mathrm{D}=10$, type IIA Supergravity}

The geometrical formulation of $\mathrm{D}=10$, type IIA SUGRA involves the following objects: the vector-like and spinor-like supervielbeins $E^{a}=d Z^{M} E_{M}{ }^{a}(Z), E_{L}^{\alpha}=d Z^{M} E_{L M}{ }^{\alpha}(Z), E_{R}^{\hat{\alpha}}=$ $d Z^{M} E_{R M}{ }^{\hat{\alpha}}(Z)$ which are one-superforms, the Lorentz-group one-superform connection $\Omega^{a b}(Z)$;

\footnotetext{
${ }^{2} \mathrm{~A}$ reason to deal with the type IIA case is the recent interest on type IIA superstings in the $A d S_{4} \times C P^{3}$ background [13] - [17].
} 
the NS-NS two-superform $B_{2}(Z)$; the dilaton superfield $\phi(Z)$; the R-R one-superform $C_{1}(Z)$ and 3 -superform and $C_{3}(Z)$. The indices $a=0,1, \ldots 9, \alpha=1, \ldots 16$ and $\hat{\alpha}=1, \ldots, 16$ ), are respectively the tangent space vector and spinor indices of $D=10$ type IIA superspace. The spinor indices are those of the Weyl-Majorana spinors with opposite chirality. $Z^{M}=\left(X^{m}, \theta_{L}^{\mu}, \theta_{R}^{\hat{\mu}}\right)$ are the superspace coordinates ${ }^{3}$.

Sometimes we shall use the notation that an upper (lower) index $\hat{\alpha}$ is written as a lower (upper) index $\alpha$ so that, for instance,

$$
E_{R}^{\hat{\alpha}} \equiv E_{R \alpha} .
$$

We shall also write $E^{\underline{\alpha}}=\left(E_{L}^{\alpha}, E_{R}^{\hat{\alpha}}\right)$ and $E^{A}=\left(E^{a}, E^{\underline{\alpha}}\right)$.

The curvatures of the superforms defined above and of the dilaton are: the torsions

$$
\begin{gathered}
T^{a}=\Delta E^{a} \equiv d E^{a}-E^{b} \Omega_{b}{ }^{a} \\
T_{L}^{\alpha}=\Delta E_{L}^{\alpha} \equiv d E_{L}{ }^{\alpha}-E_{L}{ }^{\beta} \Omega_{\beta}{ }^{\alpha} \\
T_{R}^{\hat{\alpha}}=\Delta E_{R}^{\hat{\alpha}} \equiv d E_{R}{ }^{\hat{\alpha}}-E_{R}{ }^{\hat{\beta}} \Omega_{\hat{\beta}}{ }^{\hat{\alpha}}
\end{gathered}
$$

the Lorentz curvature

$$
R^{a b}=d \Omega^{a b}-\Omega^{a}{ }_{c} \Omega^{c b}
$$

the NS-NS curvature

$$
H_{3}=d B_{2}
$$

the R-R curvatures

$$
\begin{gathered}
G_{2}=d C_{1} \\
G_{4}=d C_{3}+B_{2} d C_{1} .
\end{gathered}
$$

and

$$
F_{1}=d \phi
$$

$\Delta$ denotes the Lorentz covariant differential and $\Omega_{\underline{\beta}}^{\underline{\alpha}}=\frac{1}{4} \Omega^{a b}\left(\Gamma_{a b}\right)_{\underline{\beta}} \underline{\underline{\alpha}} \equiv\left(\Omega_{\beta}{ }^{\alpha}, \Omega_{\hat{\beta}}{ }^{\hat{\alpha}}\right)$. The torsions and curvatures (2.1)-(2.7) satisfy the free differential algebra of Bianchi identities

$$
\Delta T^{a}=-E^{b} R_{b}{ }^{a}
$$

\footnotetext{
${ }^{3}$ Letters from the middle of the alphabet denote curved indices, those from the begin of the alphabet denote tangent space, Lorentz indices. Round brackets (square brackets) will denote symmetrization (antisymmetrization) with $V^{[A} V^{B]}=\frac{1}{2}\left(V^{A} V^{B}-V^{B} V^{A}\right)$ and $V^{(A} V^{B)}=\frac{1}{2}\left(V^{A} V^{B}+V^{B} V^{A}\right)$.
} 


$$
\Delta T^{\underline{\alpha}}=-E^{\underline{\beta}} R_{\underline{\beta}}^{\underline{\alpha}}
$$

where

$$
R_{\underline{\alpha}}^{\underline{\beta}}=\frac{1}{4} R^{a b}\left(\Gamma_{a b}\right)_{\underline{\alpha}} \underline{\beta} \equiv\left(R_{L \alpha}^{\beta}, R_{R \hat{\alpha}}^{\hat{\beta}}\right) .
$$

Moreover

$$
\begin{gathered}
\Delta R^{a b}=0 \\
d H_{3}=0 \\
d G_{2}=0 \\
d G_{4}=H_{3} G_{2}
\end{gathered}
$$

(2.11) is automatically satisfied if (2.9), (2.10) hold (Dragon theorem [20]).

The field equations are obtained from the Bianchi identities after imposing suitable constraints on the torsions and curvatures [21], [22]. There is some freedom in the choice of the constraints, related to possible field redefinitions of supervielbeins and gauge superforms. We will adopt the parametrization of torsions and curvatures given in [12], [16] that, in our notation, results in the following solution of the supergravity constraints (rheonomic parametrization):

$$
\begin{gathered}
T^{a}=\frac{1}{2}\left(E_{L} \Gamma^{a} E_{L}\right)+\frac{1}{2}\left(E_{R} \Gamma^{a} E_{R}\right) \\
T_{L}^{\alpha}=\frac{1}{4}\left[E_{L}{ }^{\alpha}\left(E_{L} D_{R} \phi\right)+\frac{1}{2}\left(\Gamma^{a b}\right)^{\alpha}{ }_{\beta} E_{L}{ }^{\beta}\left(D_{R} \phi \Gamma_{a b} E_{L}\right)\right]-\frac{3}{4} E^{c}\left(\Gamma^{a b}\right)^{\alpha}{ }_{\beta} E_{L}{ }^{\beta} H_{c a b} \\
+E^{c}\left(M \Gamma_{c} E_{R}\right)^{\alpha}+E^{b} E^{c} T_{L b c}{ }^{\alpha} \\
T_{R}^{\hat{\alpha}}=\frac{1}{4}\left[E_{R}{ }^{\hat{\alpha}}\left(E_{R} D_{L} \phi\right)+\frac{1}{2}\left(\Gamma^{a b}\right)^{\hat{\alpha}}{ }_{\hat{\beta}} E_{R}{ }^{\hat{\beta}}\left(D_{L} \phi \Gamma_{a b} E_{R}\right)\right]+\frac{3}{4} E^{c}\left(\Gamma^{a b}\right)^{\hat{\alpha}}{ }_{\hat{\beta}} E_{R}{ }^{\hat{\beta}} H_{c a b} \\
-E^{c}\left(E_{L} \Gamma_{c} M\right)^{\hat{\alpha}}+E^{b} E^{c} T_{R b c}{ }^{\hat{\alpha}} \\
R^{a b}=\frac{3}{2}\left[\left(E_{L} \Gamma_{c} E_{L}\right)-\left(E_{R} \Gamma_{c} E_{R}\right)\right] H^{c a b}+2\left(E_{L} \Gamma^{[a} M \Gamma^{b]} E_{R}\right) \\
+E^{c}\left(\left(E_{L} \Theta_{R c}{ }^{a b}\right)+\left(E_{R} \Theta_{L c}{ }^{a b}\right)\right)+E^{c} E^{d} R_{c d}{ }^{a b} \\
H_{3}=-E^{a}\left[\left(E_{L} \Gamma_{a} E_{L}\right)-\left(E_{R} \Gamma_{a} E_{R}\right)\right]+E^{a} E^{b} E^{c} H_{a b c}
\end{gathered}
$$




$$
\begin{gathered}
G_{2}=-e^{\phi}\left(E_{R} E_{L}\right)-e^{\phi} E^{a}\left[\left(E_{L} \Gamma_{a} D_{L} \phi\right)-\left(E_{R} \Gamma_{a} D_{R} \phi\right)\right]+E^{a} E^{b} G_{a b} \\
G_{4}=e^{\phi} E^{a} E^{b}\left(E_{L} \Gamma_{a b} E_{R}\right)+\frac{1}{3} e^{\phi} E^{a} E^{b} E^{c}\left(\left(D_{L} \phi \Gamma_{a b c} E_{L}\right)\right. \\
\left.+\left(D_{R} \phi \Gamma_{a b c} E_{R}\right)\right)+E^{a} E^{b} E^{c} E^{d} G_{a b c d} \\
F_{1} \equiv d \phi=E_{L}^{\alpha} D_{R \alpha} \phi+E_{R}^{\hat{\alpha}} D_{L \hat{\alpha}} \phi+E^{a} D_{a} \phi
\end{gathered}
$$

where

$$
\Theta_{R / L c \mid a b}=-\left(\left(\Gamma_{a} T_{R / L b c}\right)+\left(\Gamma_{b} T_{R / L c a}\right)-\left(\Gamma_{c} T_{R / L a b}\right)\right)
$$

and $M^{\alpha \hat{\beta}} \equiv M_{\beta}^{\alpha}$ is a bispinor defined as

$$
M \equiv e^{\phi} P=e^{\phi}\left[P_{a b c d} \Gamma^{a b c d}+P_{a b} \Gamma^{a b}\right]
$$

with

$$
\begin{gathered}
P_{a b c d}=-\frac{1}{16}\left[e^{-\phi} G_{a b c d}+\frac{1}{12}\left(D_{L} \phi \Gamma_{a b c d} D_{R} \phi\right)\right] \\
P_{a b}=-\frac{1}{8}\left[e^{-\phi} G_{a b}+\frac{1}{2}\left(D_{L} \phi \Gamma_{a b} D_{R} \phi\right)\right]
\end{gathered}
$$

$D_{\underline{\alpha}} \equiv\left(D_{R \alpha}, D_{L \hat{\beta}}\right)$ and $D_{a}$ are the tangent space components of the differential $d$.

For further purposes it is convenient to display the $(0,2)$ sectors of eqs. (2.16), (2.17) (i.e. the sectors that involve products of two spinor-like superviewlbeins) by writing

$$
\begin{aligned}
& E_{L}{ }^{\beta} E_{L}{ }^{\gamma} X_{\beta \gamma}{ }^{\alpha} \equiv \frac{1}{4}\left[E_{L}{ }^{\alpha}\left(E_{L} D_{R} \phi\right)+\frac{1}{2}\left(\Gamma^{a b}\right)^{\alpha}{ }_{\beta} E_{L}{ }^{\beta}\left(D_{R} \phi \Gamma_{a b} E_{L}\right)\right] \\
& E_{R}{ }^{\hat{\beta}} E_{R}{ }^{\hat{\gamma}} X_{\hat{\beta} \hat{\gamma}}{ }^{\hat{\alpha}} \equiv \frac{1}{4}\left[E_{R}{ }^{\hat{\alpha}}\left(E_{R} D_{L} \phi\right)+\frac{1}{2}\left(\Gamma^{a b}\right)^{\hat{\alpha}}{ }_{\hat{\beta}} E_{R}{ }^{\hat{\beta}}\left(D_{L} \phi \Gamma_{a b} E_{R}\right)\right]
\end{aligned}
$$

and noting, using the Fierz identities, that the contributions of these sectors can be rewritten as

$$
\begin{aligned}
& E_{L}{ }^{\beta} E_{L}{ }^{\gamma} X_{\beta \gamma}{ }^{\alpha} \equiv-E_{L}{ }^{\alpha}\left(E_{L} D_{R} \phi\right)+\frac{1}{2}\left(E_{L} \Gamma^{a} E_{L}\right)\left(\Gamma_{a} D_{R} \phi\right)^{\alpha} \\
& E_{R}{ }^{\hat{\beta}} E_{R}{ }^{\hat{\gamma}} X_{\hat{\beta} \hat{\gamma}}^{\hat{\alpha}} \equiv-E_{R}{ }^{\hat{\alpha}}\left(E_{R} D_{L} \phi\right)+\frac{1}{2}\left(E_{R} \Gamma^{a} E_{R}\right)\left(\Gamma_{a} D_{L} \phi\right)^{\hat{\alpha}}
\end{aligned}
$$

To conclude this section let us recall some identities, which will be useful later

$$
\begin{gathered}
\Delta_{\hat{\alpha}} \Delta_{\beta} \phi=-\Delta_{\beta} \Delta_{\hat{\alpha}} \phi=-\frac{1}{2}\left(\Gamma^{c} M \Gamma_{c}\right)_{\beta \hat{\alpha}} \\
\Delta_{[\alpha} \Delta_{\beta]} \phi-\Delta_{\alpha} \phi \Delta_{\beta} \phi+\frac{1}{4}\left(\Gamma^{a b c}\right)_{\alpha \beta} H_{a b c}=0 \\
\Delta_{[\hat{\alpha}} \Delta_{\hat{\beta}]} \phi-\Delta_{\hat{\alpha}} \phi \Delta_{\hat{\beta}} \phi-\frac{1}{4}\left(\Gamma^{a b c}\right)_{\hat{\alpha} \hat{\beta}} H_{a b c}=0
\end{gathered}
$$

These identites follow by considering the sector $(0,3)$ of the Bianchi identity (2.10) (that is the terms proportial to the product of three $\left.E^{\underline{\alpha}}\right)$, using (2.18) and computing $\Delta T^{\underline{\alpha}}$ using (2.16), (2.17). $\Delta_{\underline{\alpha}}$ are the spinor-like tangent space components of the covariant diferential $\Delta$. 


\section{BRS Algebra and Pure Spinors Constraints}

As already noted, the method of the Extended Differential Algebra proposed in [12] to derive BRST transformations and pure spinor constraints is a generalization of a procedure [18], [19] well known for Yang-Mills theories, which received a large number of applications, as for instance in the descent equations for the consistent anomalies in gauge theories [23], [24], [25] or in some treatments of topological twisted theories [26], [27].

Let us describe the case of Yang-Mills theory to illustrate the method. The Yang-Mills algebra involves the connection $A \in$ LieG valued in the Lie algebra of the gauge group $\mathrm{G}$, with curvature $F=d A+A A$ and Bianchi identity $\Delta F=d F+a F-F A=0$. The extended algebra is obtained by replacing $d$ with $\hat{d}=d+\mathcal{S}$ and $A$ with $\hat{A}=A+c$ where $\mathcal{S}$ is the BRS differential and $c \in \operatorname{Lie} G$ is the ghost. The extended curvature and the extended Bianchi indetities are defined by the same algebraic relations as for the unextended ones. The extended curvature is constrained to be equal to the initial one

$$
\hat{F}=\hat{d} \hat{A}+\hat{A} \hat{A}=F
$$

so that, expanding in ghost number, eq.(3.1) is an identity in the sector with ghost number $n_{g h}=0$. In the sector with $n_{g h}=1$ one has $\mathcal{S} A=-d c-c A-A c \equiv-\Delta c$, the BRS transformation of $A$ and in the sector with $n_{g h}=2$ one has $\mathcal{S} c=-c c$, the BRS transformation of the ghost $c$.

Stora [18] calls eq. (3.1) the "Russian formula" ( see also [19] for a "superspace" derivation of this formula).

This approach can be generalized to supergravity theories. The method consists of extending the (Free) Differential Algebra of supergravity by replacing the differential $d$ with $d+\mathcal{S}$ where $\mathcal{S}$ is the (full) BRST differential and by adding to each gauge (super)-form an associated ghost. Since this extension is purely algebraic the definitions of torsions and curvatures and their parametrizations remain the same for the extended objects.

In [12] all ghosts related to bosonic gauge symmetries are set to zero (so that only the ghosts $\lambda^{\underline{\alpha}}$, related to supersymmetry, are kept), resulting in a constrained BRST algebra.

Our proposal differs from that of [12] in two respects.

The first difference is that we will keep non-zero not only the ghosts $\lambda^{\underline{\alpha}}$ related to supersymmetry but also the ghosts $\sigma_{0}, \sigma_{1}, \sigma_{2}$ and $\psi^{a b} \equiv \psi^{[a b]}$ related to the gauge transformations of $C_{1}, B_{2}, C_{3}$ and to Lorentz transformations respectively.

However we shall maintain that the ghosts $\lambda^{a}$, related to translations, vanish. The reason for taking $\lambda^{a}=0$ is that this condition is nothing else but the main constraint of the superembedding approach [28], [29]. The superembedding approach provides an alternatrive description of the Green-Schwarz approach where the k-symmetry of the Green-Schwarz formulation is reinterpreted as worldsheet supersymmetry. Its main feature is the requirment that the components of the pull-back of the supervielbeins $E^{a}$ along the odd dimensions of the superworldsheet, vanish. On the other hand, from the point of view of superembedding, the ghosts $\lambda^{A}$ associated to the supervielbeins $E^{A}$ can be identified with the pull-back of $E^{A}$ along 
a Grassmann-odd dimension, parametrized by $\eta$, of the super worldsheet, $\lambda^{A}=\partial_{\eta} Z^{M} E_{M}{ }^{A}[29]$, so that $\lambda^{a}=\partial_{\eta} Z^{M} E_{M}{ }^{a}=0$ is just the main requirement of the superembedding approach.

The second difference is motivated by the fact that the BRST transformations relevant for the pure spinor approach are those related to the ghosts $\lambda^{\underline{\alpha}}$. Therefore we split the full BRST differential $\mathcal{S}$ as

$$
\mathcal{S}=s+\delta
$$

where $s=s_{L}+s_{R}$ is the BRST differential related to the ghosts $\lambda \underline{\alpha} \equiv\left(\lambda_{L}, \lambda_{R}\right)$ and $\delta$ generates the BRST Lorentz and gauge transformations with gauge parameters given by the corresponding ghosts.

Now we can define the extended (hatted) quantities:

$$
\begin{gathered}
\hat{E}^{a}=E^{a} \\
\hat{E}_{L}^{\alpha}=E_{L}^{\alpha}+\lambda_{L}{ }^{\alpha} \\
\hat{E}_{R}{ }^{\hat{\alpha}}=E_{R}{ }^{\hat{\alpha}}+\lambda_{R}{ }^{\hat{\alpha}}
\end{gathered}
$$

As for the extension of superforms other that $E^{A}$ (which can be expressed on the basis of the supervielbeins $E^{A}$ ) there are two possibilities: or one extends the supervielbeins themselves before adding the ghosts or one keeps the supervielbeins unextended. Therefore one has

$$
\begin{gathered}
\hat{\Omega}_{a b}=\hat{E}^{C} \Omega_{C}^{a b}+\psi^{a b} \equiv \Omega^{a b}+\tilde{\psi}^{a b} \\
\hat{B}_{2}=\hat{E}^{A} \hat{E}^{B} B_{B A}+\sigma_{1} \equiv B_{2}+\tilde{\sigma}_{1} \\
\hat{C}_{1}=\hat{E}^{A} C_{A}+\sigma_{0} \equiv C_{1}+\tilde{\sigma}_{0} \\
\hat{C}_{3}=\hat{E}^{A} \hat{E}^{B} \hat{E}^{C} C_{C B A}+\sigma_{2} \equiv C_{3}+\tilde{\sigma}_{2}
\end{gathered}
$$

Of course there is a relation between the ghosts and the ghosts tilded, that follow from (3.4)(3.7), given (3.3). Indeed

$$
\begin{gathered}
\tilde{\psi}^{a b}=\psi^{a b}+i_{\lambda} \Omega^{a b}, \\
\tilde{\sigma}_{0}=\sigma_{0}+i_{\lambda} C_{1}, \\
\tilde{\sigma}_{1}=\sigma_{1}+i_{\lambda} B_{2}+\frac{1}{2} i_{\lambda}^{2} B_{2}, \\
\tilde{\sigma}_{2}=\sigma_{2}+i_{\lambda} C_{3}+\frac{1}{2} i_{\lambda}^{2} C_{3}+\frac{1}{6} i_{\lambda}^{3} C_{3} .
\end{gathered}
$$

$i_{\lambda}$ acting on a superform denotes the contraction of this superform with $\lambda^{\underline{\alpha}}$. 
One should notice that, since $B_{2}$ and $C_{3}$ are superforms of degree higher than one, $\sigma_{1}$ and $\sigma_{2}$ contain in principle ghosts of ghosts that is $\sigma_{1}=\sigma_{1}^{1}+\sigma_{0}^{2}$ and $\sigma_{2}=\sigma_{2}^{1}+\sigma_{1}^{2}+\sigma_{0}^{3}$ or $\tilde{\sigma}_{1}=\tilde{\sigma}_{1}^{1}+\tilde{\sigma}_{0}^{2}$ and $\tilde{\sigma}_{2}=\tilde{\sigma}_{2}^{1}+\tilde{\sigma}_{1}^{2}+\tilde{\sigma}_{0}^{3}$ where $\sigma_{i}^{p}$ and $\tilde{\sigma}_{i}^{p}$ have ghost number $\mathrm{p}$ and form degree i. The definition of $\tilde{\sigma}_{i}^{p}$ is obvious from (3.8), (3.9) but notice in particular that $\tilde{\sigma}_{0}^{2}$ receives a contribution also from $i_{\lambda} \sigma_{1}$ and $\tilde{\sigma}_{1}^{2}$ and $\tilde{\sigma}_{0}^{3}$ from $i_{\lambda} \sigma_{2}$ and $\frac{1}{2} i_{\lambda}^{2} \sigma_{2}$.

Using these two options, we can define in the two ways the BRST differential

$$
\hat{d}=d+s+\delta \equiv d+\tilde{s}+\tilde{\delta}
$$

In the first option realized by $\delta$, one assumes that $\delta$ induces infinitesimal Lorentz transformations with Lorentz parameter $\psi^{a b}$ acting on Lorentz tensors, Lorentz connections and relative ghosts and gauge transformations with parameters $\sigma_{i}, \mathrm{i}=(0,1,2)$, acting on $C_{1}, B_{2}$ and $C_{3}$ and relative ghosts. In particolar

$$
\begin{gathered}
\delta C_{1}+d \sigma_{0}=0=\delta B_{2}+d \sigma_{1}^{1}=0, \\
\delta C_{3}+d \sigma_{2}^{1}+B_{2} d \sigma_{0}+d \sigma_{1}^{1} C_{1}=0, \\
\delta \Omega^{a b}+\Delta \psi^{a b}=0 .
\end{gathered}
$$

Moreover

$$
\begin{gathered}
\delta \sigma_{1}^{1}+d \sigma_{0}^{2}=0=\delta \sigma_{2}^{1}+d \sigma_{1}^{2}, \\
\delta \sigma_{1}^{2}+d \sigma_{0}^{3}=0=\delta \sigma_{0}^{3}
\end{gathered}
$$

and

$$
\delta \psi^{a b}=\psi_{c}^{a} \psi^{c b}
$$

Notice that $s+\delta$ is nilpotent and anticommutes with the differential $d$. Moreover $\delta^{2}=0$ and $d \delta+\delta d=0=s \delta+\delta s$. In this case the BRST differential $s$ is nilpotent. However, just to assure nilpotency, $s$, acting on non invariant quantities, induces Lorentz transformations with parameter $i_{\lambda} \Omega^{a b}$ and gauge transformations with parameters $i_{\lambda} C_{1}, i_{\lambda} B_{2}$ and $i_{\lambda} C_{3}$.

In the second option realized by $\tilde{\delta}$, rewriting

$$
s+\delta=\tilde{s}+\tilde{\delta}
$$

as anticipated in (3.10), one assumes that $\tilde{\delta}$ acts as $\delta$ but with the Lorentz and gauge parameters $\psi^{a b}$ and $\sigma_{i}$ replaced by $\tilde{\psi}^{a b}$ and $\tilde{\sigma}_{i}$. In this case the non covariant Lorentz and gauge transformations induced by $s$ are absorbed by $\tilde{\delta}$ and $\tilde{s}$ is the covariant BRST differential. Now $\tilde{\delta}$ does not anticommute with $\tilde{s}$ and therefore $\tilde{s}$ is no longer nilpotent. For instance, acting on the Lorentz vector $V^{a}$

$$
\tilde{s}^{2} V^{a}=-\frac{1}{2} V^{b} i_{\lambda}^{2} R_{b}^{a}
$$

However, acting on Lorentz scalar and gauge invariant superfields, $\tilde{s}$ coincides with $s$ and is nilpotent.

Since $\mathrm{s}$ is always nilpotent, the nilpotent BRST charge Q, relevant to the pure spinor approach, generates the transformations induced by $s$ (not $\tilde{s}$ ). 
Now one can define the torsions and curvatures of the hatted quantities as done in (2.9)(2.15) for the unhatted ones. Since the hatted superforms have the same algebraic properties as the unhatted ones, they obey the same Bianchi identities and can be subject to the same rheonomic parametrization, namely

$$
\begin{aligned}
& \hat{T}^{a}=\hat{\Delta} \hat{E}^{a}=\frac{1}{2}\left(\hat{E}_{L} \Gamma^{a} \hat{E}_{L}\right)+\frac{1}{2}\left(\hat{E}_{R} \Gamma^{a} \hat{E}_{R}\right) \\
& \hat{H}_{3}=\hat{d} \hat{B}_{2}=-\hat{E}^{a}\left[\left(\hat{E}_{L} \Gamma_{a} \hat{E}_{L}\right)-\left(\hat{E}_{R} \Gamma_{a} \hat{E}_{R}\right)\right]+\hat{E}^{a} \hat{E}^{b} \hat{E}^{c} H_{a b c} \\
& \hat{T}_{L}^{\alpha}=\hat{\Delta} \hat{E}_{L}^{\alpha}=\frac{1}{4}\left[\hat{E}_{L}^{\alpha}\left(\hat{E}_{L} D_{R} \phi\right)+\frac{1}{2}\left(\Gamma^{a b} \hat{E}_{L}\right)^{\alpha}\left(D_{R} \phi \Gamma_{a b} \hat{E}_{L}\right)\right]-\frac{3}{4} \hat{E}^{c}\left(\Gamma^{a b} \hat{E}_{L}\right)^{\alpha} H_{c a b} \\
& +\hat{E}^{c}\left(M \Gamma_{c} \hat{E}_{R}\right)^{\alpha}+\hat{E}^{b} \hat{E}^{c} T_{L b c}^{\alpha} \\
& \hat{T}_{R}^{\hat{\alpha}}=\hat{\Delta} \hat{E}_{R}^{\hat{\alpha}}=\frac{1}{4}\left[\hat{E}_{R}^{\hat{\alpha}}\left(\hat{E}_{R} D_{L} \phi\right)+\frac{1}{2}\left(\Gamma^{a b} \hat{E}_{R}\right)^{\hat{\alpha}}\left(D_{L} \phi \Gamma_{a b} \hat{E}_{R}\right)\right]+\frac{3}{4} \hat{E}^{c}\left(\Gamma^{a b} \hat{E}_{R}\right)^{\hat{\alpha}} H_{c a b} \\
& -\hat{E}^{c}\left(\hat{E}_{L} \Gamma_{c} M\right)^{\hat{\alpha}}+\hat{E}^{b} \hat{E}^{c} T_{R b c}{ }^{\hat{\alpha}} \\
& \hat{R}^{a b}=\hat{d} \hat{\Omega}^{a b}-\hat{\Omega}^{a}{ }_{c} \hat{\Omega}^{c b}=\frac{3}{2}\left[\left(\hat{E}_{L} \Gamma^{c} \hat{E}_{L}\right)-\left(\hat{E}_{R} \Gamma^{c} \hat{E}_{R}\right)\right] H^{c a b}+2\left(\hat{E}_{L} \Gamma^{[a} M \Gamma^{b]} \hat{E}_{R}\right) \\
& +\hat{E}^{c}\left(\left(\hat{E}_{L} \Theta_{L c}{ }^{a b}\right)+\left(\hat{E}_{R} \Theta_{R c}{ }^{a b}\right)\right)+\hat{E}^{c} \hat{E}^{d} R_{c d}^{a b} \\
& \hat{G}_{2}=\hat{d} \hat{C}_{1}=-e^{\phi}\left(\hat{E}_{R} \hat{E}_{L}\right)-e^{\phi} \hat{E}^{a}\left[\left(\hat{E}_{L} \Gamma_{a} D_{L} \phi\right)-\left(\hat{E}_{R} \Gamma_{a} D_{R} \phi\right)\right]+\hat{E}^{a} \hat{E}^{b} G_{a b} \\
& \hat{G}_{4}=\hat{d} \hat{C}_{3}+\hat{B}_{2} \hat{d} \hat{C}_{1}=e^{\phi} \hat{E}^{a} \hat{E}^{b}\left(\hat{E}_{L} \Gamma_{a b} \hat{E}_{R}\right) \\
& +\frac{1}{3} e^{\phi} \hat{E}^{a} \hat{E}^{b} \hat{E}^{c}\left(\left(D_{L} \phi \Gamma_{a b c} \hat{E}_{L}\right)+\left(D_{R} \phi \Gamma_{a b c} \hat{E}_{R}\right)\right)+\hat{E}^{a} \hat{E}^{b} \hat{E}^{c} \hat{E}^{d} G_{a b c d}
\end{aligned}
$$

In the sector with zero ghost number the equations (3.12)-(3.18) reproduce the equations (2.9)$(2.15)$.

In the sector with ghost number one, following the second option, and noticing that in the left hand sides of eqs. (3.12)-(3.18) the action of $\tilde{\delta}$ cancels the tilded ghosts, these equations give

$$
\tilde{s} E^{a}=\left(\lambda_{L} \Gamma^{a} E_{L}\right)+\left(\lambda_{R} \Gamma^{a} E_{R}\right)
$$




$$
\begin{gathered}
\tilde{s} B_{2}=-2 E^{a}\left[\left(\lambda_{L} \Gamma_{a} E_{L}\right)-\left(\lambda_{R} \Gamma_{a} E_{R}\right)\right] \\
\tilde{s} E_{L}^{\alpha}=-\Delta \lambda_{L}^{\alpha}+\frac{1}{4}\left[\lambda_{L}^{\alpha}\left(E_{L} D_{R} \phi\right)+\frac{1}{2}\left(\Gamma^{a b} \lambda_{L}\right)^{\alpha}\left(D_{R} \phi \Gamma_{a b} E_{L}\right)\right] \\
+\frac{1}{4}\left[E_{L}{ }^{\alpha}\left(\lambda_{L} D_{R} \phi\right)+\frac{1}{2}\left(\Gamma^{a b} E_{L}\right)^{\alpha}\left(D_{R} \phi \Gamma_{a b} \lambda_{L}\right)\right]-\frac{3}{4} E^{c} H_{c a b}\left(\Gamma^{a b}\right)^{\alpha}{ }_{\beta} \lambda_{L}{ }^{\beta}+\left(M E^{c} \Gamma_{c} \lambda_{R}\right)^{\alpha} \\
\tilde{s} E_{R}^{\hat{\alpha}}=-\Delta \lambda_{R}{ }^{\hat{\alpha}}+\frac{1}{4}\left[\lambda_{R}{ }^{\hat{\alpha}}\left(E_{R} D_{R} \phi\right)+\frac{1}{2}\left(\Gamma^{a b} \lambda_{R}\right)^{\hat{\alpha}}\left(D_{R} \phi \Gamma_{a b} E_{R}\right)\right] \\
+\frac{1}{4}\left[E_{R}{ }^{\hat{\alpha}}\left(\lambda_{R} D_{R} \phi\right)+\frac{1}{2}\left(\Gamma^{a b} E_{R}\right)^{\hat{\alpha}}\left(D_{R} \phi \Gamma_{a b} \lambda_{R}\right)\right]+\frac{3}{4} E^{c} H_{c a b}\left(\Gamma^{a b}\right)^{\hat{\alpha}}{ }_{\hat{\beta}} \lambda_{R}{ }^{\hat{\beta}}-\left(\lambda_{L} E^{c} \Gamma_{c} M\right)^{\hat{\alpha}} \\
\tilde{s} \Omega^{a b}=3\left[\left(E_{L} \Gamma^{c} \lambda_{L}\right)-\left(E_{R} \Gamma_{c} \lambda_{R}\right)\right] H^{c a b}+2\left(\lambda_{L} \Gamma^{[a} M \Gamma^{b]} E_{R}\right)+2\left(E_{L} \Gamma^{[a} M \Gamma^{b]} \lambda_{R}\right) \\
-E^{c}\left[\left(\lambda_{L} \Theta_{L c}{ }^{a b}\right)+\left(\lambda_{R} \Theta_{R c}{ }^{a b}\right)\right] \\
\tilde{s} C_{1}=-e^{\phi}\left[\left(\lambda_{L} E_{R}\right)+\left(\lambda_{R} E_{L}\right)\right]-e^{-\phi} E^{a}\left[\left(\lambda_{L} \Gamma_{a} D_{L} \phi\right)-\left(\lambda_{R} \Gamma_{a} D_{R} \phi\right)\right] \\
\tilde{s} C_{3}=-B_{2} \tilde{s} C_{1}+e^{\phi} E^{a} E^{b}\left[\left(\lambda_{L} \Gamma_{a b} E_{R}\right)+\left(E_{L} \Gamma_{a b} \lambda_{R}\right)\right] \\
1 \\
E^{b} E^{c} e^{\phi}\left[\left(\lambda_{L} \Gamma_{a b c} D_{L} \phi\right)+\left(\lambda_{R} \Gamma_{a b c} D_{R} \phi\right)\right]
\end{gathered}
$$

Equations (3.19)-(3.25) are the BRST transformations of super vielbeins, Lorentz connection and gauge forms, derived as in [12].

The sector with ghost number two is the most interesting for our purposes since it gives the BRST trasformations of the ghosts and the ghost constraints.

Before discussing this sector it is convenient to do the mild assumption that

$$
\tilde{s} \tilde{\sigma}_{1}^{1}=0
$$

Later on we will see that this assumption is quite natural and almost implied by the formalism.

Since $\lambda^{a}=0$, from eq. (3.12) one has

$$
0=\left(\lambda_{L} \Gamma^{a} \lambda_{L}\right)+\left(\lambda_{R} \Gamma^{a} \lambda_{R}\right)
$$

With the assumption (3.26), eq. (3.13) gives

$$
0=-E^{a}\left[\left(\lambda_{L} \Gamma^{a} \lambda_{L}\right)-\left(\lambda_{R} \Gamma^{a} \lambda_{R}\right)\right]
$$

Since the vector supervielbeins are generic, equations (3.27), (3.28) imply the pure spinor constraints

$$
\left(\lambda_{L} \Gamma^{a} \lambda_{L}\right)=0=\left(\lambda_{R} \Gamma^{a} \lambda_{R}\right)
$$


Using the extended version of the definition (2.24), at the level of $n_{g h}=2$, eqs. (3.14) and (3.15) imply

$$
\begin{gathered}
\tilde{s} \lambda_{L}{ }^{\alpha}=\lambda_{L}{ }^{\beta} \lambda_{L}{ }^{\gamma} X_{\beta \gamma}{ }^{\alpha} \\
\tilde{s} \lambda_{R}{ }^{\hat{\alpha}}=\lambda_{R}{ }^{\hat{\beta}} \lambda_{R}{ }^{\hat{\gamma}} X_{\hat{\beta} \hat{\gamma}}{ }^{\hat{\alpha}}
\end{gathered}
$$

or, taking into account eqs.(2.25) and (3.29),

$$
\begin{gathered}
\tilde{s} \lambda_{L}{ }^{\alpha}=-\lambda_{L}{ }^{\alpha}\left(\lambda_{L} D_{R} \phi\right) \\
\tilde{s} \lambda_{R}{ }^{\hat{\alpha}}=-\lambda_{R}{ }^{\hat{\alpha}}\left(\lambda_{R} D_{L} \phi\right)
\end{gathered}
$$

Finally, from (3.16),(3.17) and (3.18) one has

$$
\begin{gathered}
\tilde{s} \tilde{\psi}^{a b}=\left(\lambda_{L} \Gamma^{[a} M \Gamma^{b]} \lambda_{R}\right)+\frac{3}{2}\left[\left(\lambda_{L} \Gamma_{c} \lambda_{L}\right)-\left(\lambda_{R} \Gamma_{c} \lambda_{R}\right)\right] H^{c a b} \\
\tilde{s} \tilde{\sigma}_{0}=-e^{\phi}\left(\lambda_{R} \lambda_{L}\right) \\
\tilde{s} \tilde{\sigma}_{2}^{1}+\tilde{\sigma}_{1}^{1} \tilde{s} C_{1}+\tilde{\sigma}_{0}^{2} G_{2}=e^{\phi}\left[E^{a} E^{b}\left(\lambda_{L} \Gamma_{a b} \lambda_{R}\right)+B_{2}\left(\lambda_{L} \lambda_{R}\right)\right]
\end{gathered}
$$

In [12] the constraints for the $\lambda$ 's are given by (3.27), (3.28) with $E^{a}$ restricted to the world sheet of the string together with two further constraints given by the r.h.s. of equations (3.33), (3.34). In our approach, due to the presence of the ghosts $\tilde{\sigma}_{0}, \tilde{\sigma}_{2}$ and $\tilde{\psi}^{a b}$, these last constraints are avoided as well as the constraint given by the r.h.s. of (3.32) in the absence of the ghost $\tilde{\psi}^{a b}$.

Now let us show that the assumption (3.26) is (almost) implied by the extended Free Differential Algebra under consideration.

Without the assumption (3.26), equation (3.28) becomes

$$
\tilde{s} \tilde{\sigma}_{1}^{1}=-E^{a}\left[\left(\lambda_{L} \Gamma^{a} \lambda_{L}\right)-\left(\lambda_{R} \Gamma^{a} \lambda_{R}\right)\right]
$$

Now consider the extended Bianchi identity

$$
\hat{d} \hat{G}_{4}=\hat{H}_{3} \hat{G}_{2}
$$

which is indeed automatically satisfied given (3.13), (3.17), (3.18). In the sector of ghost number four it gives

$$
e^{\phi} E^{a}\left[\left(\lambda_{L} \Gamma^{b} \lambda_{L}\right)+\left(\lambda_{R} \Gamma^{b} \lambda_{R}\right)\right]\left(\lambda_{L} \Gamma^{a b} \lambda_{R}\right)=e^{\phi} E^{a}\left[\left(\lambda_{L} \Gamma^{a} \lambda_{L}\right)-\left(\lambda_{R} \Gamma^{a} \lambda_{R}\right)\right]\left(\lambda_{L} \lambda_{R}\right)
$$

Then, from (3.27) and assuming

$$
\left(\lambda_{L} \lambda_{R}\right) \neq 0,
$$


equations (3.28) and (3.26) follow. The other possible solution of $(3.36),\left(\lambda_{L} \lambda_{R}\right)=0$, is the one relevant to the $\mathrm{D}=11$, supermembrane [30], [31]. Equation (3.36) is quite obvious since it is nothing else but the Fierz identity in eleven dimensions

$$
(\Gamma \underline{a b})_{(\underline{\alpha} \underline{\beta}}\left(\Gamma_{\underline{b}}\right)_{\underline{\gamma} \underline{\delta})}=0,
$$

reduced to 10 dimensions and saturated with $\lambda^{\underline{\alpha}}, \lambda \underline{\beta}, \lambda \underline{\underline{\alpha}}, \lambda^{\underline{\delta}}$

Given the equations (3.19)-(3.34) one should verify the nilpotency of the BRST charge $Q$ acting on these superforms and ghosts. This can be done systematically starting from the relevant Bianchi identities but in the most of the cases it is very easy to perform the checks directly.

We conclude this section by deriving a useful property of the left handed and right handed BRS differential of the bispinor $P^{\alpha \hat{\beta}}$. Using (3.28), equation $(3.32)$ can be written as $(\tilde{s}+\tilde{\delta}) \tilde{\psi}^{a b}=$ $\tilde{\psi}^{a}{ }_{c} \tilde{\psi}^{c b}+\left(\lambda_{L} \Gamma^{[a} M \Gamma^{b]} \lambda_{R}\right)$. Then the nilpotency of $(s+\delta)=(\tilde{s}+\tilde{\delta})$ yields the identity

$$
\tilde{s}\left(\lambda_{L} \Gamma^{[a} e^{\phi} P \Gamma^{b]} \lambda_{R}\right)=0
$$

where $e^{\phi} P^{\alpha \hat{\beta}}$ is defined in (2.23). Taking into account (3.31), this equation gives

$$
\left(\lambda_{L} \Gamma^{[a}\left[\left(\lambda_{L}{ }^{\alpha} \Delta_{\alpha} P+\lambda_{R}{ }^{\hat{\alpha}} \Delta_{\hat{\alpha}} P\right)\right] \Gamma^{b]} \lambda_{R}\right)=0
$$

Equation (3.38) also follows from the Bianchi identity

$$
\hat{\Delta} \hat{R}^{a b}=0
$$

in the sector with ghost number three.

If one defines

$$
\begin{aligned}
& \tilde{s}_{L} P^{\beta \hat{\gamma}} \equiv \lambda_{L}{ }^{\alpha} \Delta_{\alpha} P^{\beta \hat{\gamma}}=\lambda_{L}{ }^{\alpha} C_{L \alpha}{ }^{\beta \hat{\gamma}} \\
& \tilde{s}_{R} P^{\beta \hat{\gamma}} \equiv \lambda_{R}{ }^{\hat{\alpha}} \Delta_{\hat{\alpha}} P^{\beta \hat{\gamma}}=\lambda_{R}{ }^{\hat{\alpha}} C_{R \hat{\alpha}}{ }^{\beta \hat{\gamma}}
\end{aligned}
$$

equation (3.38) implies

$$
\begin{aligned}
& C_{L \alpha}{ }^{\beta \hat{\gamma}}=\delta_{\alpha}{ }^{\beta} C_{L}{ }^{\hat{\gamma}}+\frac{1}{4}\left(\Gamma^{a b}\right)_{\alpha}{ }^{\beta} C_{L a b}{ }^{\hat{\gamma}} \\
& C_{R \hat{\alpha}}^{\beta \hat{\gamma}}=\delta_{\hat{\alpha}}^{\hat{\gamma}} C_{R}^{\beta}+\frac{1}{4}\left(\Gamma^{a b}\right)_{\hat{\alpha}}^{\hat{\gamma}} C_{R a b}{ }^{\beta}
\end{aligned}
$$

Now note that a generic matrix $Y_{\alpha}{ }^{\beta}$ can be decomposed as follows:

$$
Y_{\alpha}{ }^{\beta}=Y^{(0)} \delta_{\alpha}{ }^{\beta}+Y_{a b}^{(2)}\left(\Gamma^{a b}\right)_{\alpha}{ }^{\beta}+Y_{a b c d}^{(4)}\left(\Gamma^{a b c d}\right)_{\alpha}{ }^{\beta} .
$$

If the term $Y_{a b c d}^{(4)}$ is absent we shall say that $Y_{\alpha}{ }^{\beta}$ is Lorentz and Weyl valued or in short $L W$ valued. The same is valid for the matrix $Y_{\hat{\alpha}}^{\hat{\beta}}$. For instance, according to (3.39), $C_{L \alpha}{ }^{\beta \hat{\gamma}}$ and $C_{R \hat{\alpha}}{ }^{\beta \hat{\gamma}}$ are $\mathrm{LW}$-valued with respect to the indices $\alpha, \beta$ and $\hat{\alpha}, \hat{\gamma}$ respectively.

This result will be important in the next section to assure the consistency of the pure spinor action. 


\section{Pure Spinor Action}

In this section we derive the pure spinor action, in two different ways that will be described in the subsections 4.1 and 4.2 respectively. As a result we recover the action first obtained in [7].

Before doing that, some preliminary considerations are in order. The ghosts $\sigma^{\prime}$ s and $\psi$ do not appear in the action, while one must add to the superspace coordinates $Z^{M}$ and the ghosts $\lambda \underline{\alpha}$ the antighosts

$$
\omega_{\underline{\alpha}}=\left(\omega_{R \alpha}, \omega_{L \hat{\alpha}}\right)
$$

with ghost number $n_{g}=-1$, which are the conjugate momenta of $\lambda^{\underline{\alpha}}$, and the fields

$$
d_{\underline{\alpha}}=\left(d_{R \alpha}, d_{L \hat{\alpha}}\right)
$$

that involve the conjugate momenta of $Z^{M}$ and are essentially the BRS partners of $\omega_{\underline{\alpha}}$. From the worldsheet point of view, $\omega_{R}, d_{R}$ and $\omega_{L}, d_{L}$ are right-handed and left-handed chiral fields respectively.

Since, as a consequence of the pure spinor constraints, $\lambda^{\underline{\alpha}}$ contains $11+11$ degree of freedom, also $\omega_{\underline{\alpha}}$ should contain $11+11$ independent components. This is realized by assuming that the pure spinor action is invariant under the $\omega$-gauge symmetry

$$
\delta^{(\omega)} \omega_{R / L}=\Lambda_{R / L}^{a}\left(\Gamma_{a} \lambda_{L / R}\right)
$$

where $\Lambda_{R / L}^{a}$ are gauge parameters.

The $d_{\underline{\alpha}}$ appear in the nilpotent BRS charge ${ }^{4}$

$$
Q=Q_{L}+Q_{R}=\oint \lambda \underline{\underline{\alpha}} d_{\underline{\alpha}}
$$

that is

$$
\begin{aligned}
Q_{L} & =\oint\left(\lambda_{L} d_{R}\right), \\
Q_{R} & =\oint\left(\lambda_{R} d_{L}\right) .
\end{aligned}
$$

under which the action must be invariant. In order to specify $Q$ and prove its nilpotency one needs the expression for $d_{\underline{\alpha}}$. As has already been noted, $Q$ generates the transformations induced by the BRS differential $s$ and a suggestion to obtain an ansatz for $d_{\underline{\alpha}}$ comes from the action of $s$ on superfields and ghosts. In particular from (3.30) one has

$$
s \lambda^{\underline{\alpha}}=\lambda^{\underline{\beta}} \lambda \underline{\underline{\gamma}}\left[\Omega_{\underline{\beta \gamma}} \underline{\underline{\alpha}}+X_{\underline{\beta \gamma}} \underline{\underline{\alpha}}\right]
$$

Since the BRS trasformations of $\lambda \underline{\alpha}$ do not vanish, $d_{\underline{\alpha}}$ must contain terms linear in $\omega$ in order to reproduce (4.2). Therefore a general form of $d_{\underline{\alpha}}$ is expected to be

$$
d_{R \alpha}=d_{R \alpha}^{(0)}+\left(\Omega_{\alpha \beta}^{\gamma}+X_{\alpha \beta}^{\gamma}\right) \omega_{R \gamma} \lambda_{L}^{\beta}+\Omega_{\alpha \hat{\beta}} \hat{\gamma}_{L \hat{\gamma}} \lambda_{R}{ }^{\hat{\beta}}
$$

\footnotetext{
${ }^{4}$ Here and in the following $\oint$ denotes $\int d \sigma_{+}$or $\int d \sigma_{-}$according to the case where $\sigma_{ \pm}$are worldsheet light-cone coordinates.
} 


$$
d_{L \hat{\alpha}}=d_{L \hat{\alpha}}^{(0)}+\left(\Omega_{\hat{\alpha} \hat{\beta}}^{\hat{\gamma}}+X_{\hat{\alpha} \hat{\beta}}^{\hat{\gamma}}\right) \omega_{L \hat{\gamma}} \lambda_{R}{ }^{\hat{\beta}}+\Omega_{\hat{\alpha} \beta}{ }^{\gamma} \omega_{R \gamma} \lambda_{L}{ }^{\beta}
$$

where $d^{(0)}$ does not depend on $\omega$ and $\lambda$ and $\Omega_{\underline{\alpha} \underline{\underline{\gamma}}}$ are the tangent space spinorial components of the Lorentz connection. There is an ambiguity in the form of $X_{\beta \gamma} \underline{\underline{\alpha}}$ since (4.2) specify only the component of this superfield which is symmetric in $\underline{\beta}, \underline{\gamma}$ and modulo the pure spinor constraints. A convenient choice of $X_{\underline{\alpha} \beta} \underline{\underline{\gamma}} \equiv\left(X_{\alpha \beta}{ }^{\gamma}, X_{\hat{\alpha} \hat{\beta}} \hat{\gamma}\right)$ that reproduces (4.2) (i.e (3.31)) and is LW-valued in $\underline{\beta}, \underline{\gamma}$ is

$$
\begin{array}{r}
X_{\alpha \beta}{ }^{\gamma}=\frac{1}{4}\left[\delta_{\beta}^{\gamma} D_{R \alpha} \phi+\frac{1}{2}\left(\Gamma^{a b}\right)_{\beta}^{\gamma}\left(\Gamma_{a b} D_{R} \phi\right)_{\alpha}\right] \\
X_{\hat{\alpha} \hat{\beta}}^{\hat{\gamma}}=\frac{1}{4}\left[\delta_{\hat{\beta}}^{\hat{\gamma}} D_{R \hat{\alpha}} \phi+\frac{1}{2}\left(\Gamma^{a b}\right)_{\hat{\beta}}^{\hat{\gamma}}\left(\Gamma_{a b}\right)_{\hat{\alpha}}^{\hat{\delta}} D_{R \hat{\delta}} \phi\right]
\end{array}
$$

$d_{\underline{\alpha}}^{(0)}$ acting on superfields induces the tangent space derivatives $D_{\underline{\alpha}}$ and

$$
\left\{Q,\left(d_{R / L \underline{\alpha}}^{(0)}\right\}=-\left(E_{\mp}^{a} \Gamma_{a} \lambda_{L / R}\right)_{\underline{\alpha}}+2 \lambda \underline{\underline{\beta}}\left(\Omega_{(\underline{\beta} \underline{\alpha})^{\underline{\gamma}}}+X_{(\underline{\beta} \underline{\alpha}}\right)^{\underline{\gamma}}\right) d_{\underline{\gamma}}
$$

where $E_{ \pm}^{a}$ are the pullbacks of the vector-like vielbeins $E^{a}$ on the worldsheet.

With this expression for $d_{\underline{\alpha}}$ the BRS charge is

$$
Q=\oint \lambda \underline{\underline{\alpha}}\left[d_{\underline{\alpha}}^{(0)}+\left(\Omega_{\underline{\alpha} \underline{\underline{\gamma}}}+X_{\underline{\alpha} \underline{\underline{\beta}}}\right) \omega_{\underline{\gamma}} \lambda \underline{\underline{\beta}}\right]
$$

and

$$
Q^{2}=-\oint \lambda^{\underline{\alpha}} \lambda \underline{\underline{\beta}} \lambda \underline{\underline{\gamma}} \tilde{R}_{\underline{\alpha} \underline{\beta}} \underline{\underline{\delta}} \omega_{\underline{\delta}}
$$

where $\tilde{R}_{L \alpha}{ }^{\beta}$ and $\tilde{R}_{R \hat{\alpha}}{ }^{\hat{\beta}}$ are the curvatures of the Lorentz connections $\tilde{\Omega}_{L \alpha}{ }^{\beta}=\Omega_{\alpha}{ }^{\beta}+E_{L}^{\gamma} X_{\gamma \alpha}{ }^{\beta}$ and $\tilde{\Omega}_{R \hat{\alpha}}^{\hat{\beta}}=\Omega_{\hat{\alpha}}^{\hat{\beta}}+E_{R}^{\hat{\gamma}} X_{\hat{\gamma} \hat{\alpha}}^{\hat{\beta}}$

By an explicit computation of the l.h.s. of this equation and using the identities (2.26) and (2.27) one can verify that $Q$ is indeed nilpotent. From the expression for $Q$ one can also compute the BRS transformations of $\omega_{\underline{\alpha}}$ and $d_{\underline{\alpha}}$

$$
\begin{gathered}
s \omega_{\underline{\alpha}}=-d_{\underline{\alpha}}-\lambda \underline{\beta}\left(\Omega_{\underline{\underline{\beta}} \underline{\underline{\alpha}}}+X_{\underline{\beta} \underline{\alpha}}\right) \omega_{\underline{\gamma}} \\
s d_{\underline{\alpha}}=-\left(E_{\mp}^{a} \Gamma_{a}\right)_{\underline{\alpha} \underline{\beta}} \lambda^{\underline{\beta}}+2 \lambda \underline{\underline{\beta}} \tilde{R}_{\underline{\alpha} \underline{\beta} \underline{\underline{\gamma}}} \lambda \underline{\gamma} \omega_{\underline{\delta}}+\lambda^{\underline{\beta}}\left(\Omega_{\underline{\beta} \underline{\underline{\alpha}}} \underline{\gamma}+X_{\underline{\beta} \underline{\alpha}}\right) d_{\underline{\gamma}}
\end{gathered}
$$

The covariant form of equation (4.5) is

$$
\tilde{s} \omega_{\underline{\alpha}}=-d_{\underline{\alpha}}-\lambda \underline{\underline{\beta}} X_{\underline{\beta} \underline{\alpha}} \underline{\underline{ }} \omega_{\underline{\gamma}},
$$

that is

$$
\begin{gathered}
\tilde{s}_{L / R} \omega_{R / L \underline{\alpha}}=-d_{R / L \underline{\alpha}}-\lambda \frac{\beta}{L}{ }_{R} X_{\underline{\beta} \underline{\alpha}} \underline{\underline{\gamma}} \omega_{R / L \underline{\gamma}} \\
\tilde{s}_{R / L} \omega_{R / L \underline{\alpha}}=0
\end{gathered}
$$


Eq. (4.7) contains a term proportional to $\left(\omega_{R / L} \Gamma_{a b c d} \lambda_{L / R}\right)$ which is non invariant under (4.1). It is useful to remark that in the covariant BRS transformation of $e^{\phi} \omega_{R / L}$ this ill-behaved term is removed. Indeed, using the identity

$$
\omega_{\underline{\alpha}} \lambda^{\underline{\beta}}=\frac{1}{16}\left[\delta_{\underline{\alpha}} \underline{\beta}(\omega \lambda)-\frac{1}{2}\left(\Gamma^{a b}\right)_{\underline{\alpha}} \underline{\beta}\left(\omega \Gamma_{a b} \lambda\right)+\frac{1}{24}\left(\Gamma^{a b c d}\right)_{\underline{\alpha}} \underline{\beta}\left(\omega \Gamma_{a b c d} \lambda\right)\right],
$$

one has

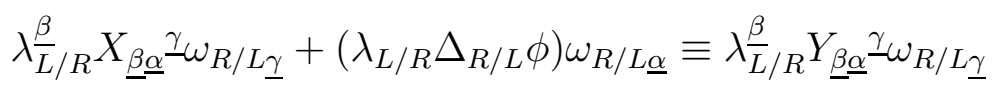

where

$$
\begin{gathered}
Y_{\beta \alpha}^{\gamma}=\frac{1}{4}\left[3 \delta_{\beta}^{\gamma} D_{\alpha} \phi-\frac{1}{2}\left(\Gamma_{a b}\right)_{\beta}^{\gamma}\left(\Gamma_{a b} D_{R} \phi\right)_{\alpha}\right]=Y_{\alpha \beta}{ }^{\gamma} \\
Y_{\hat{\beta} \hat{\alpha}}^{\hat{\gamma}}=\frac{1}{4}\left[3 \delta_{\hat{\beta}}^{\hat{\gamma}} D_{\hat{\alpha}} \phi-\frac{1}{2}\left(\Gamma_{a b}\right)_{\hat{\beta}}^{\hat{\gamma}}\left(\Gamma_{a b} D_{L} \phi\right)_{\hat{\alpha}}\right]=Y_{\hat{\alpha} \hat{\beta}}^{\hat{\gamma}}
\end{gathered}
$$

are $\mathrm{LW}$-valued superfields with respect to $\beta, \gamma$ and $\hat{\beta}, \hat{\gamma}$ respectively and

$$
\tilde{s}_{L / R}\left(e^{\phi} \omega_{R / L \underline{\alpha}}\right)=-d_{R / L \underline{\alpha}}+\lambda \frac{\beta}{L / R} \omega_{R / L} \underline{\gamma} Y_{\underline{\beta}} \underline{\gamma}
$$

It is instructive to compute the action of $Q^{2}$ on $\lambda^{\underline{\alpha}}, d_{\underline{\alpha}}$ and $\omega_{\underline{\alpha}}$.

For $\lambda$ one can confirm that $Q$ applied to $\lambda^{\underline{\alpha}}$ is nilpotent since $s^{2} \lambda \underline{\alpha}=\lambda \underline{\underline{\delta}} \lambda \underline{\underline{\beta}} \lambda \underline{\underline{\gamma}} \tilde{R}_{\underline{\delta} \beta \gamma} \underline{\alpha}=0$.

At first sight, this is not true for $d_{\underline{\alpha}}$ since taking the BRST differential of (4.6), after some algebra, one obtains

$$
s^{2} d_{R / L \underline{\alpha}}=-\left(\Gamma^{a} \lambda_{L / R}\right)_{\underline{\alpha}}\left(\lambda_{R / L} \Gamma_{a}\right)_{\underline{\beta}}\left[E_{\bar{\mp} R / L}^{\underline{\beta}} \pm\left(s_{L / R}\left(M_{R / L} \omega_{R / L}\right)^{\underline{\beta}}\right]\right.
$$

where $M_{L}=M, M_{R}$ is the transpose of $M$ and $\mathrm{M}$ is defined in (2.23). However, if we assume that $E_{\mp R / L}$ satisfy the field equations

$$
\left(E_{ \pm R / L}\right)^{\underline{\alpha}} \pm s_{L / R}\left(M_{R / L}^{\underline{\alpha} \underline{\beta}} \omega_{R / L \underline{\beta}}\right)=0
$$

$s^{2} d_{R / L}$ vanishes on shell. Afterward we shall prove that these field equations are indeed satisfied as variations of the pure spinor action with respect to $d_{\alpha}$.

However $s^{2} \omega$ does not vanish since from (4.5) and (4.6) one has

$$
s^{2} \omega_{R / L}=\left(E_{\mp}^{a} \Gamma_{a} \lambda_{L / R}\right)
$$

This failure of nilpotency is a consequence of the $\omega$-gauge transformation (4.1). Indeed $s^{2}$, acting on $\omega$, vanishes only modulo this gauge transformation.

Therefore eq (4.12) is consistent and one can proceed to derive the pure spinor action living with eq. (4.12). This is done in subsection 4.1 following [12]

However, even if (4.12) is consistent, one could be disappointed by the fact that the square of a nilpotent charge gives a non vanishing result acting on some object. It is possible to avoid this result by fixing the $\omega$-gauge symmetry and using the so called Y-formalism [29], [32] - [35]. In this formalism one can construct the pure spinor action in a way which is more on line with the original proposal considered in [8] for the heterotic string. This is done in subsection 4.2. 


\subsection{Derivation of the pure spinor action}

The general method to construct pure spinor superstring actions in generic backgrounds is the following. One starts from the Green-Schwarz action $I_{G S}$ in conformal gauge, computes its BRS variation and then adds a "gauge fixing" action $I_{g f}$ given by the (left-handed and right-handed) BRS variation of a suitable "gauge fermion" ( a functional with ghost number $n_{g}=-1$ ) such that the total action is BRS invariant.

$I_{g f}$ has to satisfy two conditions:

i) Its BRS variation must cancel the variation of the Green-Schwarz term.

ii) It must be invariant under the gauge transformations (4.1) i.e. its dependence on $\omega_{R / L}$ should involve only the terms $\left(\omega_{R / L} \lambda_{L / R}\right),\left(\omega_{R / L} \Gamma_{a b} \lambda_{L / R}\right)$ and $\left(\omega_{R / L} d \lambda_{L / R}\right)$.

The Green-Schwarz action is

$$
I_{G S}=\frac{1}{2} \int\left[E_{+}^{a} \eta_{a b} E_{-}^{b}+B_{+-}\right]
$$

where $B_{+-}$is the pullback of the NS-NS superform $B_{2}$.

The BRS variation of $I_{G S}$ is

$$
s I_{G S}=\int\left[\left(\lambda_{L} E_{-}^{a} \Gamma_{a} E_{+L}\right)+\left(\lambda_{R} E_{+}^{a} \Gamma_{a} E_{-R}\right)\right]
$$

A term which fulfils the condition i) is

$$
I_{g f}^{(0)}=-\int s\left[\left(E_{+L} \omega_{R}\right)+\left(E_{-R} \omega_{L}\right)\right]
$$

Indeed, taking into account that $s^{2}$ is non vanishing only when acts on $\omega_{L / R}$ and using (4.12), one can see that $s I_{g f}^{(0)}$ cancels the variation of $I_{G S}$. However this term cannot be the whole story since when $\tilde{s}_{R / L}$ acts on $E_{ \pm L / R}$, the term

$$
I_{g f}^{(1)}=-\int\left[\left(\omega_{R} M E_{+}^{a} \Gamma_{a} \lambda_{R}\right)-\left(\lambda_{L} E_{-}^{a} \Gamma_{a} M \omega_{L}\right)\right]
$$

arises, which is incompatible with the condition ii). Therefore, following [12], we replace $I_{g f}^{(0)}$ with

$$
I_{g f}^{\prime(0)}=I_{g f}^{(0)}-I_{g f}^{(1)}=-\int\left[s_{L}\left(E_{+L} \omega_{R}\right)+s_{R}\left(E_{-R} \omega_{L}\right)\right]
$$

$I_{g f}^{(1)}$ can be rewritten as

$$
I_{g f}^{(1)}=-\int\left(s_{R}^{2}-s_{L}^{2}\right)\left(\omega_{R} M \omega_{L}\right)
$$

This expression suggests to add the term

$$
I_{g f}^{(2)}=1 / 2 \int\left(s_{L} s_{R}-s_{R} s_{L}\right)\left(\omega_{R} M \omega_{L}\right)=\int s_{L} s_{R}\left(\omega_{R} M \omega_{L}\right)
$$


in order to cancel the variation of $I_{g f}^{(1)}$. As a result, the "gauge fixing" action is

$$
I_{g f}=I_{g f}^{(0)}-I_{g f}^{(1)}+I_{g f}^{(2)}=-\int\left[s_{L}\left(E_{+L} \omega_{R}\right)+s_{R}\left(E_{-R} \omega_{L}\right)\right]+\int s_{L} s_{R}\left(\omega_{R} M \omega_{L}\right)
$$

This expression for the "gauge fixing" action has been proposed in [12] and shown to be BRS invariant. In fact the variation of $I_{g f}^{(0)}$ cancels the variation of the G-S action, as already noted, and

$$
s I_{g f}^{(1)}=s I_{g f}^{(2)} .
$$

Indeed the variation of $I_{g f}^{(1)}$ is

$$
s I_{g f}^{(1)}=\int\left(s_{L} s_{R}^{2}-s_{R} s_{L}^{2}\right)\left(\omega_{R}\left(e^{\phi} P\right) \omega_{L}\right)
$$

and the variation of $I_{g f}^{(2)}$ is

$$
s I_{g f}^{(2)}=\int\left(s_{L}^{2} s_{R}+s_{R} s_{L} s_{R}\right)\left(\omega_{R}\left(e^{\phi} P\right) \omega_{L}\right)
$$

Since $\left(s_{L} s_{R}+s_{R} s_{L}\right)$ always vanishes, the r.h.s.'s of these two equations are equal.

Now let us compute $I_{g f}$.

Using $(3.21),(3.22)$ and $(4.5)$ one gets for $I_{g f}^{(0)}-I_{g f}^{(1)}$

$$
I_{g f}^{(0)}-I_{g f}^{(1)}=\int\left[\left(E_{+L} d_{R}\right)+\left(E_{-R} d_{L}\right)+\left(\omega_{R} \Delta_{+}^{(L)} \lambda_{L}\right)+\left(\omega_{L} \Delta_{-}^{(R)} \lambda_{R}\right)\right]
$$

where

$$
\left(\Delta^{(L / R)} \lambda_{L / R}\right)^{\underline{\alpha}}=d \lambda^{\underline{\alpha}}-\lambda \underline{\beta} \Omega_{(L / R)} \underline{\beta}^{\underline{\alpha}}-\frac{1}{4} \lambda \underline{\alpha}\left(E_{L / R} D_{L / R} \phi\right)
$$

and $\Omega_{(L / R) \underline{\beta}^{\underline{\alpha}}}$ is defined as

$$
\Omega_{(L / R) \underline{\beta}}^{\underline{\alpha}}=\frac{1}{4}\left(\Gamma_{a b}\right)_{\underline{\beta}}^{\underline{\alpha}}\left(\Omega^{a b}+\frac{1}{2}\left(E_{L / R} \Gamma^{a b} D_{L / R} \phi\right) \mp 3 E^{c} H_{c}^{a b}\right)
$$

Note that the second term in the variation of $\omega_{L / R}$ (see equations (4.5), (4.3)) is essential for the consistency of the result since it cancels the terms $1 / 4\left[\left(\omega_{R / L} E_{L / R}\right)\left(\lambda_{L / R} D_{L / R} \phi\right)+\right.$ $\left.1 / 2\left(\omega_{R / L} \Gamma^{a b} E_{L / R}\right)\left(D_{L / R} \phi \Gamma_{a b} \lambda_{L / R}\right)\right]$ coming from the variation of $E_{L / R}$ (equations $(3.21),(3.22)$ ). In the absence of this term in the r.h.s. of (4.5) the result would be inconsistent with the condition ii). This is an important consistency check of the ansatz (4.3) for $X_{\underline{\beta \gamma}}$.

To compute $I_{g f}^{(2)}$ it is convenient to use the first expression for $I_{g f}^{(2)}$ in (4.17) to get

$$
\begin{gathered}
I_{g f}^{(2)}=-\int\left(\tilde{s}_{L}\left(\omega_{R} e^{\phi}\right) P e^{-\phi} \tilde{s}_{R}\left(\omega_{L} e^{\phi}\right)\right)-\int\left(\tilde{s}_{L}\left(\omega_{R} e^{\phi}\right)\left(\tilde{s}_{R} P\right) \omega_{L}\right)-\int\left(\omega_{R}\left(\tilde{s}_{L} P\right) \tilde{s}_{R}\left(\omega_{L} e^{\phi}\right)\right) \\
+\frac{1}{2} \int e^{\phi}\left(\omega_{R \alpha}\left(\left(\tilde{s}_{R} \tilde{s}_{L}-\tilde{s}_{L} \tilde{s}_{R}\right) P^{\alpha \hat{\alpha}}\right) \omega_{L \hat{\alpha}}\right)
\end{gathered}
$$




$$
+\frac{1}{2} \int e^{\phi}\left[\left(\tilde{s}_{R} \tilde{s}_{L} \omega_{R \alpha}+\left(\tilde{s}_{R} \tilde{s}_{L} \phi\right) \omega_{R \alpha}\right) P^{\alpha \hat{\alpha}} \omega_{L \hat{\alpha}}-\omega_{R \alpha} P^{\alpha \hat{\alpha}}\left(\tilde{s}_{L} \tilde{s}_{R} \omega_{L \hat{\alpha}}+\left(\tilde{s}_{L} \tilde{s}_{R} \phi\right) \omega_{L \hat{\alpha}}\right)\right]
$$

The terms involving $\tilde{s}_{R / L} \tilde{s}_{L / R} \phi$ in the last integral arise to avoid double counting.

The first three terms in the r.h.s. of (4.22) are consistent with the condition ii) since the monomials $\lambda \underline{\alpha} \omega_{\underline{\beta}}$ are saturated by LW-valued superfields. In fact

$$
\begin{gathered}
I_{g f}^{(a)} \equiv \int\left(\tilde{s}_{L}\left(\omega_{R} e^{\phi}\right) P e^{\phi} \tilde{s}_{R}\left(\omega_{L} e^{\phi}\right)\right)=\int\left(d_{R \alpha}-\lambda_{L}{ }^{\beta} \omega_{R \gamma} Y_{\beta \alpha}{ }^{\gamma}\right) e^{\phi} P^{\alpha \hat{\alpha}}\left(d_{L \hat{\alpha}}-\lambda_{R}{ }^{\hat{\beta}} \omega_{L \hat{\gamma}} Y_{\hat{\beta} \hat{\alpha}}^{\hat{\gamma}}\right) \\
I_{g f}^{(b)} \equiv \int\left(\tilde{s}_{L}\left(\omega_{R} e^{\phi}\right)\left(\tilde{s}_{R} P\right) \omega_{L}\right)=-\left(d_{R \alpha}-\lambda_{L}{ }^{\beta} \omega_{R \gamma} Y_{\beta \alpha}{ }^{\gamma}\right) e^{\phi} C_{R \hat{\beta}}{ }^{\alpha \hat{\gamma}} \lambda_{R}{ }^{\hat{\beta}} \omega_{L \hat{\gamma}}
\end{gathered}
$$

and

$$
I_{g f}^{(c)} \equiv \int\left(\omega_{R}\left(\tilde{s}_{L} P\right) \tilde{s}_{R}\left(\omega_{L} e^{\phi}\right)\right)=-\lambda_{L}{ }^{\beta} \omega_{R \alpha} e^{\phi} C_{L \beta}^{\alpha \hat{\alpha}}\left(d_{L \hat{\alpha}}-\lambda_{R}{ }^{\hat{\beta}} \omega_{L \hat{\gamma}} Y_{\hat{\beta} \hat{\alpha}}^{\hat{\gamma}}\right)
$$

The last two integrals in (4.22), that is,

$$
I_{g f}^{(d)}=\frac{1}{2} \int e^{\phi}\left(\omega_{R \alpha}\left(\left(\tilde{s}_{R} \tilde{s}_{L}-\tilde{s}_{L} \tilde{s}_{R}\right) P^{\alpha \hat{\alpha}}\right) \omega_{L \hat{\alpha}}\right)
$$

and

$$
I_{g f}^{(e)}=\frac{1}{2} \int e^{\phi}\left[\left(\tilde{s}_{R} \tilde{s}_{L} \omega_{R \alpha}+\left(\tilde{s}_{R} \tilde{s}_{L} \phi\right) \omega_{R \alpha}\right) P^{\alpha \hat{\alpha}} \omega_{L \hat{\alpha}}-\omega_{R \alpha} P^{\alpha \hat{\alpha}}\left(\tilde{s}_{L} \tilde{s}_{R} \omega_{L \hat{\alpha}}+\left(\tilde{s}_{L} \tilde{s}_{R} \phi\right) \omega_{L \hat{\alpha}}\right)\right]
$$

are potentially dangerous since both give contributions that violate condition ii). Luckily these contributions cancel each other. This is proved in the Appendix where the LW-valued contributions of the last two integrals are computed. The result is

$$
I_{g f}^{(d)}+I_{g f}^{(e)}=\int e^{\phi} \omega_{R \beta} \lambda_{L}{ }^{\alpha} \omega_{L \hat{\gamma}} \lambda_{R}{ }^{\hat{\delta}} C_{\alpha \hat{\delta}}^{\beta \hat{\gamma}}+\int e^{\phi} \omega_{R \beta} \lambda_{L}{ }^{\alpha} \omega_{L \hat{\gamma}} \lambda_{R} \hat{\delta} \tilde{C}_{\alpha \hat{\delta}} \beta \hat{\gamma}
$$

where

$$
C_{\alpha \hat{\delta}}^{\beta \hat{\gamma}}=\Pi_{\alpha \sigma}^{\beta \tau}\left(\Delta_{[\tau} \Delta_{\hat{\tau}]} P^{\sigma \hat{\sigma}}\right) \Pi_{\hat{\sigma} \hat{\delta}}^{\hat{\gamma} \hat{\gamma}}
$$

and

$$
\tilde{C}_{\beta \hat{\delta}} \gamma \hat{\gamma}=e^{\phi} \Pi_{\beta \tau} \gamma \sigma\left[R_{\sigma \hat{\sigma} \rho}^{\tau} P^{\rho \hat{\tau}}-R_{\sigma \hat{\sigma} \hat{\rho}}^{\hat{\tau}} P^{\tau \hat{\rho}}+\left(\Gamma^{c} P \Gamma_{c}\right)_{\sigma \hat{\sigma}} P^{\tau \hat{\tau}}\right] \Pi_{\hat{\tau} \hat{\delta}}^{\hat{\sigma} \hat{\gamma}}
$$

where

$$
\Pi_{\alpha \sigma}^{\beta \tau}=\delta_{\alpha}{ }^{\beta} \delta_{\sigma}{ }^{\tau}-\frac{1}{16.4 !}\left(\Gamma^{a b c d}\right)_{\alpha}{ }^{\beta}\left(\Gamma_{a b c d}\right)_{\sigma}{ }^{\tau}
$$

is the projector that projects on the LW-valued component of a superfield $Y_{\tau}^{\sigma}$ and $\Pi_{\hat{\sigma} \hat{\delta}} \hat{\tau}^{\hat{\gamma}}$ is defined in a similar way in terms of the hatted quantities. 
Adding to the Green-Schwarz action (4.13) the contributions of $I_{g f}$ which are given in (4.19), (4.23), (4.24), (4.25), and (4.28) one obtains the pure spinor action for type IIA superstring in a general background:

$$
\begin{gathered}
I=\int\left[\frac{1}{2}\left(E_{+}^{a} E_{-a}+B_{+-}\right)+E_{+L}^{\alpha} d_{R \alpha}+E_{-R}^{\hat{\alpha}} d_{L \hat{\alpha}}\right. \\
+\omega_{R \alpha}\left(\Delta_{+}^{(L)} \lambda_{L}\right)^{\alpha}+\omega_{L \hat{\alpha}}\left(\Delta_{-}^{(R)} \lambda_{R}\right)^{\hat{\alpha}}-d_{R \alpha} M^{\alpha \hat{\beta}} d_{L \hat{\beta}} \\
\left.+d_{R \gamma} e^{\phi} \tilde{C}^{\gamma \hat{\alpha}}{ }_{\hat{\beta}} \omega_{L \hat{\alpha}} \lambda_{R}{ }^{\hat{\beta}}+\omega_{R \beta} \lambda_{L}^{\alpha} e^{\phi} \tilde{C}_{\alpha}^{\hat{\gamma} \beta} d_{L \hat{\gamma}}-\omega_{R \alpha} \lambda_{L}^{\beta} S_{\beta \hat{\delta}}^{\alpha \hat{\gamma}} \omega_{L \hat{\gamma}} \lambda_{R}^{\hat{\delta}}\right]
\end{gathered}
$$

where $\Delta_{ \pm}^{(L / R)}$ are the pullbacks of the covariant differentials defined in $(4.20),(4.21) . \tilde{C}^{\gamma \hat{\alpha}}{ }_{\hat{\beta}}$, $\tilde{C}^{\hat{\gamma} \alpha}$ and $S_{\beta \hat{\delta}}^{\alpha \hat{\gamma}}$ are given by

$$
\begin{gathered}
\tilde{C}_{\hat{\beta}}^{\gamma \hat{\alpha}}=C_{R \hat{\beta}}{ }^{\hat{\alpha} \gamma}+P^{\gamma \hat{\gamma}} Y_{\hat{\beta} \hat{\gamma}}{ }^{\hat{\alpha}} \\
\tilde{C}^{\hat{\gamma} \alpha}{ }_{\beta}=C_{L \beta}{ }^{\alpha \hat{\gamma}}+P^{\gamma \hat{\gamma}} Y_{\beta \gamma}{ }^{\alpha} \\
S_{\beta \hat{\delta}}^{\alpha \hat{\gamma}}=C_{\beta \hat{\gamma}}^{\alpha \hat{\gamma}}+\tilde{C}_{\beta \hat{\gamma}}^{\alpha \hat{\gamma}}+Y_{\beta \gamma}{ }^{\alpha} P^{\gamma \hat{\beta}} Y_{\hat{\delta} \hat{\beta}} \hat{\gamma}
\end{gathered}
$$

where $P, C_{R \hat{\beta}}^{\hat{\alpha} \gamma}, C_{L \beta}^{\alpha \hat{\gamma}}, Y_{\hat{\beta} \hat{\gamma}}^{\hat{\alpha}}, Y_{\beta \gamma}{ }^{\alpha}, C_{\beta \hat{\delta}}^{\alpha \hat{\gamma}}$ and $\tilde{C}_{\beta \hat{\delta}}^{\alpha \hat{\gamma}}$ are defined in the equations (2.23), (3.39), (4.8), (4.29), (4.30) so that all the superfields in (4.31) are given explicitly in terms of the components of torsions and curvatures, or more specifically in terms of $P, \phi$ and their (covariant) derivatives.

The action is manifestly invariant under BRS transformations as well as under the gauge tranformation (4.1) of $\omega$. Moreover the field equations obtained from the action (4.31) varying $d_{\underline{\alpha}}$ are

$$
\begin{aligned}
& E_{+L}^{\alpha}=-e^{\phi}\left(P_{L}^{\alpha \hat{\beta}} d_{L \hat{\beta}}-\tilde{C}^{\alpha \hat{\beta}} \lambda_{R}^{\hat{\gamma}} \omega_{L \hat{\beta}}\right) \\
& E_{-R}^{\hat{\alpha}}=e^{\phi}\left(P_{R}^{\hat{\alpha} \beta} d_{R \beta}-\tilde{C}_{\gamma}^{\hat{\alpha} \beta} \lambda_{L}^{\gamma} \omega_{R \beta}\right) .
\end{aligned}
$$

They are identical to equations (4.11) and assure the on shell nilpotency of $s$ acting on $d_{\underline{\alpha}}$. The action (4.31) is precisely the action first obtained in [7].

\subsection{Alternative derivation of the action. The Y-formalism}

Y-formalism.

If the equal time Poisson Brackets (ETPB's) among $\omega$ and $\lambda$ are the canonical ones, the ETPB's among $\omega_{R / L}$ and $\left(\lambda_{L / R} \Gamma^{a} \lambda_{L / R}\right)$ do not vanish:

$$
\left\{\omega(\sigma)_{R / L \underline{\alpha}},\left(\lambda\left(\sigma^{\prime}\right)_{L / R} \Gamma^{a} \lambda\left(\sigma^{\prime}\right)_{L / R}\right)\right\}=2\left(\Gamma^{a} \lambda_{L / R}\left(\sigma^{\prime}\right)\right)_{\underline{\alpha}} \delta\left(\sigma^{\prime}-\sigma\right)
$$


The constraint $\left(\lambda_{L / R} \Gamma^{a} \lambda_{L / R}\right)=0$ generates the gauge transformation (4.1) of $\omega_{R / L}$, but in the pure spinor approach this constraint is assumed to hold in a strong sense and therefore equation (4.33) is unsatisfactory. This problem can be avoided [1], [8] by assuming the following ETPB among $\omega$ and $\lambda$ :

$$
\left\{\omega_{\underline{\alpha}}(\sigma), \lambda \underline{\beta}\left(\sigma^{\prime}\right)\right\}=\delta\left(\sigma-\sigma^{\prime}\right)\left[\delta_{\underline{\alpha}} \underline{\beta}-K_{\underline{\alpha}} \underline{\beta}\right]
$$

where $K_{\underline{\alpha}}^{\underline{\beta}} \equiv\left(K_{L \alpha}{ }^{\beta}, K_{R \hat{\alpha}} \hat{\beta}^{\hat{\beta}}\right)$ are the projectors

$$
\begin{aligned}
& K_{L \alpha}{ }^{\beta}=\frac{1}{2}\left(\Gamma^{a} \lambda_{L}\right)_{\alpha}\left(Y_{R} \Gamma_{a}\right)^{\beta} \\
& K_{R \hat{\alpha}}^{\hat{\beta}}=\frac{1}{2}\left(\Gamma^{a} \lambda_{R}\right)_{\hat{\alpha}}\left(Y_{L} \Gamma_{a}\right)^{\hat{\beta}}
\end{aligned}
$$

with

$$
Y_{R / L \underline{\alpha}}=\frac{V_{R / L \underline{\alpha}}}{\left(V_{R / L} \lambda_{L / R}\right)}
$$

so that

$$
\left(Y_{R / L} \lambda_{L / R}\right)=1
$$

If one chooses $V_{R / L}$ constant, $K_{\underline{\alpha}} \underline{\beta}$ breaks Lorentz invariance. Moreover it is singular at $\left(V_{L / R} \lambda_{L / R}\right)=0$.

In the case of IIA superstrings one can avoid the breaking of Lorentz invariance by choosing

$$
Y_{R / L}=\frac{\lambda_{R / L}}{\left(\lambda_{R} \lambda_{L}\right)} .
$$

In the following we shall adopt this choice. With this choice, if one defines

$$
K_{\beta}^{\alpha}=\frac{1}{2}\left(\Gamma^{a} \lambda_{R}\right)^{\alpha} \frac{1}{\left(\lambda_{L} \lambda_{R}\right)}\left(\lambda_{L} \Gamma_{a}\right)_{\beta},
$$

$K_{R}$ and $K_{L}$ are transposed to each other, and

$$
K_{\beta}^{\alpha}=\left(K_{R}\right)^{\alpha}{ }_{\beta}
$$

Even if now the Lorentz invariance is preserved, $K$ is still singular when $\left(\lambda_{R} \lambda_{L}\right)=0$. In any case these deseases - breaking of Lorentz invariance and/or singular nature of $K$ - are innocuous since, as we shall see, any dependence of $K$ will be absent in the final results.

Gauge fixing.

One can gauge fix the $\omega$-gauge symmetry (4.1) by requiring

$$
\left(\omega_{R / L} \Gamma^{a} \lambda_{R / L}\right)=0 .
$$

Using the projector $K$, this gauge fixing condition is equivalent to

$$
\left(K \omega_{L}\right)=0=\left(\omega_{R} K\right)
$$


or

$$
\omega_{R \alpha}=\omega_{R \beta}(1-K)^{\beta}{ }_{\alpha} \quad \omega_{L}^{\alpha}=(1-K)^{\alpha}{ }_{\beta} \omega_{L}^{\beta}
$$

which are consistent with (4.34). Also notice that

$$
\left(K \lambda_{L}\right)^{\alpha}=0=\left(\lambda_{R} K\right)_{\alpha}
$$

and

$$
\left(\lambda_{L} \Gamma^{a}(1-K)\right)_{\alpha}=0=\left((1-K) \Gamma^{a} \lambda_{R}\right)^{\alpha}
$$

Equation (4.40), toghether with (4.38) implies that $\omega_{R / L}$ have vanishing ETPB's with the constraint (3.29). Moreover, since $\operatorname{Tr} K_{R / L}=5, K_{L}$ and $K_{R}$ project on five-dimensional subspaces of the 16-dimensional spinorial spaces and therefore each of the ghosts $\lambda_{L}, \lambda_{R}, \omega_{R}$ and $\omega_{L}$ has eleven components. The fields $d_{\underline{\alpha}}$ can be splitted as

$$
\begin{gathered}
d_{R \alpha}^{\top}=\left(d_{R}(1-K)\right)_{\alpha} \\
d_{L}^{\top \alpha}=\left((1-K) d_{L}\right)^{\alpha} \\
d_{R \alpha}^{\perp}=\left(d_{R} K\right)_{\alpha} \\
d_{L}^{\perp \alpha}=\left(K d_{L}\right)^{\alpha} .
\end{gathered}
$$

Only $d_{R / L}^{\top}$ appears in the BRS charge $Q$ so that $d_{R / L}^{\top}$ are the BRST partners of $\omega_{R / L}$. With these definitions, the BRST transformations of $\omega_{\underline{\alpha}}, d_{\underline{\alpha}}^{\top}, d_{\underline{\alpha}}^{\perp}$ can be obtained by projecting (4.5) and (4.6) on the subspaces spanneds by the projectors $\frac{K}{K}$ and $(1-K)$. In particular

$$
\begin{gathered}
\tilde{s} d_{R \alpha}^{\perp}=-\left[\left(\lambda_{L} E_{-}^{a} \Gamma_{a}\right)_{\gamma}-2\left(\lambda_{L} \lambda_{R}\right)\left(\omega_{R} M\right)_{\gamma}+\left(\lambda_{L} D_{R} \phi\right) d_{R \gamma}^{\perp}-\lambda_{L}^{\beta} Y_{\beta \gamma}{ }^{\delta} d^{\perp}{ }_{R \delta}\right] K_{\alpha}^{\gamma} \\
\tilde{s} d_{L}^{\perp \alpha}=-K_{\gamma}^{\alpha}\left[\left(E_{+}^{a} \Gamma_{a} \lambda_{R}\right)^{\gamma}+2\left(\lambda_{L} \lambda_{R}\right)\left(M \omega_{L}\right)^{\gamma}+\left(\lambda_{R} D_{L} \phi\right) d^{\perp \gamma}-\lambda_{R}^{\hat{\beta}} Y_{\hat{\beta} \hat{\tau}}^{\hat{\sigma}} d_{L \hat{\sigma}}^{\perp} \delta^{\hat{\tau} \gamma}\right]
\end{gathered}
$$

Projecting (4.10), (4.12) with $(1-K)$, one has

$$
s^{2} d_{R / L}^{\top}=0=s^{2} \omega_{R / L}
$$

Moreover

$$
\begin{aligned}
& s^{2} d_{R \alpha}^{\perp}=-\left(\lambda_{R} \lambda_{L}\right)\left(E_{-R}+\tilde{s}_{L}\left(\omega_{R} M\right)\right)_{\gamma} K^{\gamma}{ }_{\alpha} \\
& s^{2} d_{L}^{\perp \alpha}=-\left(\lambda_{R} \lambda_{L}\right) K_{\gamma}^{\alpha}\left(E_{+L}-\tilde{s}_{R}\left(M \omega_{L}\right)\right)^{\gamma}
\end{aligned}
$$

The right hand sides of (4.43) vanish on shell if $E_{\mp R / L}$ satisfy the field equations (4.11). It follows from (4.42), (4.43) that now s is nilpotent acting on any field or ghost.

Derivation of the Action 
In this formalism the strategy to derive the pure spinor action is similar to [8]. Add to the Green-Schwarz action a new $K$-dependent term $I_{(K)}$ such that $I_{G S}+I_{(K)}$ is BRST invariant. Then add the "gauge fixing" term $I_{g f}=s \int F$ to cancel the dependence on $K$ in the total action. The "gauge fermion" $F$ is a local functional with $n_{g h}=-1$. Since $s$ is always nilpotent, $I_{g f}$ is automatically BRS invariant.

A possible choice of $I_{(K)}$ is

$$
I_{(K)}=-\int\left[\left(d_{R} K E_{+L}\right)-\left(E_{-R} K d_{L}\right)\right]-\int\left(d_{R} K M K d_{L}\right)-2 \int\left(\lambda_{L} \lambda_{R}\right)\left(\omega_{R} M_{R} K M_{L} \omega_{L}\right)
$$

Indeed

$$
\begin{aligned}
\tilde{s}\left[\left(d_{R} K E_{+L}\right)-\left(E_{-R} K d_{L}\right)\right]= & {\left[\left(\lambda_{L} E_{-}^{a} \Gamma_{a} E_{+L}\right)+\left(\lambda_{R} E_{+}^{a} \Gamma_{a} E_{-R}\right)\right]-2\left(\lambda_{L} \lambda_{R}\right)\left[\left(\omega_{R} M K E_{+L}\right)-\left(E_{-R} K M \omega_{L}\right)\right] } \\
& +\left[\left(d_{R} K M E_{+}^{a} \Gamma_{a} \lambda_{R}\right)-\left(\lambda_{L} \Gamma_{a} E_{-}^{a} M K d_{L}\right)\right]
\end{aligned}
$$

$\tilde{s}\left(d_{R} K M K d_{L}\right)=-\left[\left(d_{R} K M E_{+}^{a} \Gamma_{a} \lambda_{L}\right)-\left(\lambda_{R} \Gamma_{a} E_{-}^{a} M K d_{L}\right)\right]-2\left(\lambda_{L} \lambda_{R}\right)\left[\left(\omega_{R} M K M K d_{L}\right)+\left(d_{R} K M K M \omega_{L}\right)\right]$

and

$$
\begin{gathered}
2 \tilde{s}\left[\left(\lambda_{L} \lambda_{R}\right)\left(\omega_{R} M K M \omega_{L}\right)\right]=-2\left(\lambda_{L} \lambda_{R}\right)\left[\left(\omega_{R} M K M(1-K) d_{L}\right)+\left(d_{R}(1-K) M K M \omega_{L}\right)\right] \\
\left.+2\left(\lambda_{L} \lambda_{R}\right) e^{\phi}\left[\omega_{R}\left(\lambda_{L}^{\alpha} \tilde{C}_{L \alpha}\right) K M \omega_{L}\right)+\left(\omega_{R} M K\left(\lambda_{R}^{\hat{\alpha}} \tilde{C}_{R \hat{\alpha}}\right) \omega_{L}\right)\right]
\end{gathered}
$$

so that

$$
s I_{G F}+s I_{(K)}=0
$$

modulo the field equations (4.32).

Then choosing

$$
\begin{gathered}
I_{g f}=s \int\left[\left(d_{R} K M \omega_{L}\right)-\left(\omega_{R} M K d_{L}\right)\right]-s \int\left[\left(\omega_{R} E_{+L}\right)+\left(E_{-R} \omega_{L}\right)\right] \\
+\frac{1}{2} s \int\left[\left(\tilde{s}_{R}-\tilde{s}_{L}\right)\left(\omega_{R} M \omega_{L}\right)\right]
\end{gathered}
$$

one can verify that the total action

$$
I=I_{G S}+I_{(K)}+I_{g f}
$$

reproduces the pure spinor action (4.31). 


\section{Conclusion}

To summarize, in this paper, generalizing the method of Extended Differential Algebra, proposed in [12], we have shown that it is possible to start from a geometrical formulation of Type IIA supergravity (rheonomic parametrization of torsions and curvatures) and derive the standard pure spinor constraints, the nilpotency of the BRST charge, and the BRST invariant action of the pure spinor superstrings formulation. The pure spinor constraints follow from the requirement that the ghosts $\lambda^{a}$, related to the vector-like supervielbeins, vanish (similar to the superembedding constraint) together with the mild assumption that $\left(\lambda_{L} \lambda_{R}\right)$ does not generically vanish.

In a sense this reverses the pattern followed in [7] where, starting from the nilpotency of the BRST charge and the most general BRST invariant action (or, equivalently, the holomorphicity properties of the BRST currents), a consistent set of on shell supergravity constraints is derived.

The results that we obtain are equivalent to those of [7] modulo the different choice of the supergravity constraints. However, the supergravity constraints from which we start (and the superfields which appear in the final action) differ from those derived in [7] at most by a redefinition of supervielbeins, superconnections, gauge superforms and the dilaton. The fact that in [7] the structure group is very large (it involves three independent local Lorentz groups, for vector, left-handed and right-handed spinors and two independent local Weyl groups for left-handed and right-handed spinors) should not deceive. Indeed, as shown in [7], this large gauge symmetry must be gauge fixed and reduced to a single local Lorentz invariance in order to cancel some spurious superfields in torsion components. However the form of the left-handed and right-handed Lorentz and (gauge fixed) Weyl connections remain different and this is also an a posteriori result of our approach ( see (4.20) and (4.21)).

The pure spinor action has been derived in two ways. In particular the second derivation is a generalization to the case of IIA superstring of a procedure first proposed in [8] for the heterotic string. An advantage of this method is that, once the Green-Schwarz action is modified by the addition of suitable K-dependent terms in order to promote its k-symmetry to a pure-spinor BRS symmetry, the remaining step to get the pure spinor action is a standard BRS-like gauge fixing procedure i.e. the addition of a BRS exact, local action.

The rheonomic parametrization that we have adopted in this paper is that considered in [12]. However, since all the consistent parametrizations are equivalent (in the sense specified

above) it is quite evident that the procedure described in this paper can be used starting with any consistent rheonomic parametrization.

\section{Acknowledgments}

I would like to thank D. Sorokin for valuable discussions and advices and I. Bandos and I. Oda for useful comments. 


\section{Appendix. Computation of $I_{g f}^{(d)}+I_{g f}^{(e)}$}

In this Appendix we study the integrals $I_{g f}^{(d)}$ and $I_{g f}^{(e)}$ defined in (4.26) and (4.27) and show that the contributions of these integrals that violate the $\omega$-gauge invariance cancel each other.

To compute $I_{g f}^{(d)}$ let us consider the left-handed and right-handed BRS variations of eqs.(3.39)

$$
\begin{aligned}
& \tilde{s}_{R} \tilde{s}_{L} P^{\beta \hat{\gamma}} \equiv \lambda_{L}{ }^{\alpha} \lambda_{R}{ }^{\hat{\delta}} \Delta_{\hat{\delta}} C_{L \alpha}{ }^{\beta \hat{\gamma}} \\
& \tilde{s}_{L} \tilde{s}_{R} P^{\beta \hat{\gamma}} \equiv \lambda_{L}{ }^{\alpha} \lambda_{R}{ }^{\hat{\delta}} \Delta_{\alpha} C_{R \hat{\delta}} \beta \hat{\gamma}
\end{aligned}
$$

It follows from (3.40) that $\Delta_{\hat{\delta}} C_{L \alpha}{ }^{\beta \hat{\gamma}}$ and $\Delta_{\alpha} C_{R \hat{\delta}}{ }^{\beta \hat{\gamma}}$ are LW-valued with respect to $\alpha, \beta$ and $\hat{\delta}, \hat{\gamma}$ respectively, so that we can write

$$
\frac{1}{2} \int e^{\phi} \omega_{R \alpha}\left(\left(\tilde{s}_{R} \tilde{s}_{L} P^{\alpha \hat{\alpha}}\right) \omega_{L \hat{\alpha}}\right)=\int e^{\phi} \omega_{R \beta} \lambda_{L}^{\alpha} \omega_{L \hat{\gamma}} \lambda_{R}^{\hat{\delta}}\left[C_{L \alpha \hat{\delta}}^{\beta \hat{\gamma}}+\Xi_{L \alpha \hat{\delta}}^{\beta \hat{\gamma}}\right]
$$

and

$$
\left.\frac{1}{2} \int e^{\phi} \omega_{R \alpha}\left(\tilde{s}_{L} \tilde{s}_{R} P^{\alpha \hat{\alpha}}\right) \omega_{L \hat{\alpha}}\right)=\int e^{\phi} \omega_{R \beta} \lambda_{L}{ }^{\alpha} \omega_{L \hat{\gamma}} \lambda_{R}^{\hat{\delta}}\left[C_{R \alpha \hat{\delta}}^{\beta \hat{\gamma}}+\Xi_{R \alpha \hat{\delta}}^{\beta \hat{\gamma}}\right]
$$

where $C_{L / R \alpha \hat{\delta}}^{\beta \hat{\gamma}}$ are LW-valued both in the indices $\alpha, \beta$ and $\hat{\delta}, \hat{\gamma}$ whereas $\Xi_{L \alpha \hat{\delta}}^{\beta \hat{\gamma}}$ is the contribution which is LW-valued in $\alpha, \beta$ but not in $\hat{\delta}, \hat{\gamma}$, the latter being proportional to $\left(\Gamma^{a b c d}\right)_{\hat{\delta}} \hat{\gamma}^{\text {. On }}$ the other hand $\Xi_{R \alpha \hat{\delta}}^{\beta \hat{\gamma}}$ is LW-valued in $\hat{\delta}, \hat{\gamma}$ but not in $\alpha, \beta$ which is proportional to $\left(\Gamma^{a b c d}\right)_{\alpha}{ }^{\beta}$.

Therefore

$$
\begin{gathered}
I_{g f}^{(d)}=\frac{1}{2} \int e^{\phi} \omega_{R \alpha}\left(\left(\tilde{s}_{R} \tilde{s}_{L}-\tilde{s}_{L} \tilde{s}_{R}\right) P^{\alpha \hat{\alpha}}\right) \omega_{L \hat{\alpha}} \\
=\int e^{\phi} \omega_{R \beta} \lambda_{L}{ }^{\alpha} \omega_{L \hat{\gamma}} \lambda_{R} \hat{\delta}\left[C_{\alpha \hat{\delta}}^{\beta \hat{\gamma}}+\left(\Xi_{R \alpha \hat{\delta}}^{\beta \hat{\gamma}}-\Xi_{L \alpha \hat{\delta}} \beta \hat{\gamma}\right)\right]
\end{gathered}
$$

where

$$
C_{\alpha \hat{\delta}}^{\beta \hat{\gamma}} \equiv C_{L \alpha \hat{\delta}}^{\beta \hat{\gamma}}-C_{R \alpha \hat{\delta}}^{\beta \hat{\gamma}}=\Pi_{\alpha \sigma}^{\beta \tau}\left(\Delta_{[\tau} \Delta_{\hat{\tau}]} P^{\sigma \hat{\sigma}}\right) \Pi_{\hat{\sigma} \hat{\delta}}^{\hat{\delta} \hat{\gamma}}
$$

$\Pi_{\alpha \sigma}{ }^{\beta \tau}$ and $\Pi_{\hat{\sigma} \hat{\delta}}^{\hat{\gamma} \hat{\gamma}}$ being the projectors that project on the well-behaved components of superfields $Y_{\alpha}{ }^{\beta}$ and $Y_{\hat{\delta}}^{\hat{\gamma}}$ respectively. $C_{\alpha \hat{\delta}} \beta \hat{\gamma}$ are LW-valued both in $\alpha, \beta$ and $\hat{\delta}, \hat{\gamma}$.

To compute $\Xi_{R / L}$ let us consider, instead of (A.5), the integral

$$
\begin{gathered}
\frac{1}{2} \int e^{\phi} \omega_{R \alpha}\left(\left(\tilde{s}_{R} \tilde{s}_{L}+\tilde{s}_{L} \tilde{s}_{R}\right) P^{\alpha \hat{\alpha}}\right) \omega_{L \hat{\alpha}}=\int e^{\phi} \omega_{R \beta} \lambda_{L}{ }^{\alpha} \omega_{L \hat{\gamma}} \lambda_{R} \hat{\delta}\left[C_{L \alpha \hat{\delta}}^{\beta \hat{\gamma}}+C_{R \alpha \hat{\delta}}^{\beta \hat{\gamma}}\right. \\
\left.\quad+\left(\Xi_{R \alpha \hat{\delta}}^{\beta \hat{\gamma}}+\Xi_{L \alpha \hat{\delta}}^{\beta \hat{\gamma}}\right)\right]=-\int e^{\phi} \omega_{R \beta} \lambda_{L}{ }^{\alpha} \omega_{L \hat{\gamma}} \lambda_{R} \hat{\delta}\left[R_{\alpha \hat{\delta} \tau}{ }^{\beta} P^{\tau \hat{\gamma}}+R_{\alpha \hat{\delta} \hat{\sigma}} \hat{\gamma} P^{\beta \hat{\sigma}}\right]
\end{gathered}
$$


where the last equality follows taking into account the action of $\tilde{s}^{2}$ on Lorentz valued fields (see (3.11)). By performing in (A.7) a gauge transformation of $\omega_{L \hat{\gamma}}$ i.e.by replacing $\omega_{L \hat{\gamma}}$ with $\Lambda_{c}\left(\lambda_{R} \Gamma^{c}\right)_{\hat{\gamma}}$ only $\Xi_{L}$ survives and is determined unambiguosly from the last identity in (A.7). The same can be repeated for $\Xi_{R}$ by replaciung $\omega_{R \beta}$ with $\Lambda_{c}\left(\lambda_{L} \Gamma^{c}\right)_{\beta}$.

Then to compute $I^{(e)}$ let us write

$$
I_{g f}^{(e)}=I_{g f}^{(e 1)}-I_{g f}^{(e 2)}
$$

where

$$
\begin{gathered}
I_{g f}^{(e 1)}=\frac{1}{2} \int e^{\phi}\left(\tilde{s}_{R} \tilde{s}_{L} \omega_{R \alpha}+\left(\tilde{s}_{R} \tilde{s}_{L} \phi\right) \omega_{R \alpha}\right) P^{\alpha \hat{\alpha}} \omega_{L \hat{\alpha}} \equiv \lambda_{L}{ }^{\beta} \omega_{R \gamma} \lambda_{R}{ }^{\hat{\delta}} \omega_{L \hat{\gamma}} X_{(L) \beta \hat{\delta}} \hat{\gamma} \\
I_{g f}^{(e 2)}=\frac{1}{2} \int e^{\phi}\left(\omega_{R \alpha} P^{\alpha \hat{\alpha}}\left(\tilde{s}_{L} \tilde{s}_{R} \omega_{L \hat{\alpha}}+\left(\tilde{s}_{L} \tilde{s}_{R} \phi\right) \omega_{L \hat{\alpha}}\right)\right) \equiv \lambda_{L}{ }^{\beta} \omega_{R \gamma} \lambda_{R}{ }^{\hat{\delta}} \omega_{L \hat{\gamma}} X_{(R) \beta \hat{\delta}} \hat{\gamma}
\end{gathered}
$$

It follows from (4.9) that $X_{(L)}$ is LW-valued in $\beta, \gamma$ and $X_{(R)}$ is LW-valued in $\hat{\delta}, \hat{\gamma}$ so that we can write

$$
X_{L / R \beta \hat{\delta}} \gamma \hat{\gamma}=\tilde{C}_{L / R \beta \hat{\delta}} \gamma \hat{\gamma}+\Psi_{L / R \beta \hat{\delta}} \gamma \hat{\gamma}
$$

and

$$
I_{g f}^{(e)}=I_{g f}^{(e 1)}-I_{g f}^{(e 2)}=\lambda_{L}{ }^{\beta} \omega_{R \gamma} \lambda_{R}{ }^{\hat{\delta}} \omega_{L \hat{\gamma}}\left(\tilde{C}_{\beta \hat{\delta}} \gamma \hat{\gamma}+\Psi_{R \beta \hat{\delta}} \hat{\gamma}-\Psi_{L \beta \hat{\delta}} \gamma \hat{\gamma}\right)
$$

where $\tilde{C} \equiv \tilde{C}_{L}-\tilde{C}_{R}$ is LW-valued both in $\beta, \gamma$ and in $\hat{\delta}, \hat{\gamma}$ and $\Psi_{L \beta \hat{\delta}} \gamma \hat{\gamma}\left(\Psi_{R \beta \hat{\delta}} \gamma \hat{\gamma}\right)$ is LW-valued in $(\beta, \gamma)$ but not in $(\hat{\delta}, \hat{\gamma})($ in $(\hat{\delta}, \hat{\gamma})$ but not in $(\beta, \gamma))$. Since $\tilde{s}_{R} \omega_{R}=0=\tilde{s}_{L} \omega_{L}, I_{g f}^{(e 1)}$ and $I_{g f}^{(e 2)}$ can be rewritten as

$$
\begin{aligned}
& I_{g f}^{(e 1)}=\frac{1}{2} \int e^{\phi}\left[\left(\tilde{s}_{R} \tilde{s}_{L}+\tilde{s}_{L} \tilde{s}_{R}\right) \omega_{R \alpha}+\left(\tilde{s}_{R} \tilde{s}_{L} \phi\right) \omega_{R \alpha}\right] P^{\alpha \hat{\alpha}} \omega_{L \hat{\alpha}} \\
& =\int e^{\phi}\left[\lambda_{L}{ }^{\alpha} \omega_{R \beta} \lambda_{R}{ }^{\hat{\delta}} \omega_{L \hat{\gamma}} R_{\alpha \hat{\delta} \tau}^{\beta} P^{\tau \hat{\gamma}}+\left(\tilde{s}_{R} \tilde{s}_{L} \phi\right) \omega_{R \alpha} P^{\alpha \hat{\alpha}} \omega_{L \hat{\alpha}}\right] \\
& \left.I_{g f}^{(e 2)}=\frac{1}{2} \int e^{\phi} \omega_{R \alpha} P^{\alpha \hat{\alpha}}\left[\left(\tilde{s}_{L} \tilde{s}_{R}+\tilde{s}_{R} \tilde{s}_{L}\right) \omega_{L \hat{\alpha}}\right)+\left(\tilde{s}_{R} \tilde{s}_{L} \phi\right) \omega_{L \hat{\alpha}}\right] \\
& =\int e^{\phi}\left[\lambda_{L}{ }^{\alpha} \omega_{R \beta} \lambda_{R}{ }^{\hat{\delta}} \omega_{L \hat{\gamma}} R_{\alpha \hat{\delta} \hat{\gamma}}^{\hat{\gamma}} P^{\beta \hat{\tau}}+\omega_{R \alpha} P^{\alpha \hat{\alpha}}\left(\tilde{s}_{L} \tilde{s}_{R} \phi\right) \omega_{L \hat{\alpha}}\right]
\end{aligned}
$$

Now, instead of $I_{g f}^{(e 1)}-I_{g f}^{(e 2)}$, let us consider $I_{g f}^{(e 1)}+I_{g f}^{(e 2)}$.

$$
I_{g f}^{(e 1)}+I_{g f}^{(e 2)}=\frac{1}{2} \int e^{\phi}\left[\left(\left(\tilde{s}_{R} \tilde{s}_{L}+\tilde{s}_{L} \tilde{s}_{R}\right) \omega_{R \alpha}\right) P^{\alpha \hat{\alpha}} \omega_{L \hat{\alpha}}+\left(\omega_{R \alpha} P^{\alpha \hat{\alpha}}\left(\tilde{s}_{L} \tilde{s}_{R}+\tilde{s}_{L} \tilde{s}_{R}\right) \omega_{L \hat{\alpha}}\right)\right]
$$


so that

$$
I_{g f}^{(e 1)}+I_{g f}^{(e 2)}=\int e^{\phi} \lambda_{L}{ }^{\alpha} \omega_{R \beta} \lambda_{R}{ }^{\hat{\delta}} \omega_{L \hat{\gamma}}\left[R_{\alpha \hat{\delta} \tau}{ }^{\beta} P^{\tau \hat{\gamma}}+R_{\alpha \hat{\delta} \hat{\sigma}} \hat{\gamma} P^{\beta \hat{\sigma}}\right]
$$

From this equation one can compute $\Psi_{R / L \beta \hat{\delta}} \gamma \hat{\gamma}$ following the same argument used before to compute $\Xi_{R / L \beta \hat{\delta}} \hat{\gamma}$. Comparing the right hand sides of (A.7) and (A.12) one finally concludes that

$$
\Psi_{R / L \beta \hat{\delta}}^{\gamma \hat{\gamma}}=-\Xi_{R / L \beta \hat{\delta}} \gamma \hat{\gamma}
$$

so that

$$
I_{g f}^{(d)}+I_{g f}^{(e)}=-\frac{1}{2} \int e^{\phi} \lambda_{L}{ }^{\beta} \omega_{R \gamma} \lambda_{R} \hat{\delta}_{L \hat{\gamma}}\left(C_{\beta \hat{\delta}} \gamma \hat{\gamma}+\tilde{C}_{\beta \hat{\delta}} \gamma \hat{\gamma}\right)
$$

Moreover, from (A.10), (A.11) and taking into account (2.26) it follows that

$$
\tilde{C}_{\beta \hat{\delta}}^{\gamma \hat{\gamma}}=e^{\phi} \Pi_{\beta \tau}^{\gamma \sigma}\left[R_{\sigma \hat{\sigma} \rho}^{\tau} P^{\rho \hat{\tau}}-R_{\sigma \hat{\sigma} \hat{\rho}}^{\hat{\tau}} P^{\tau \hat{\rho}}+\left(\Gamma^{c} P \Gamma_{c}\right)_{\sigma \hat{\sigma}} P^{\tau \hat{\tau}}\right] \Pi_{\hat{\gamma} \hat{\delta}}^{\hat{\gamma} \hat{\gamma}}
$$

\section{References}

[1] N. Berkovits, JHEP 0004 (2000) 018, hep-th/0001035.

[2] N. Berkovits, JHEP 0409 (2004) 047, hep-th/0406055.

[3] N. Berkovits and B.C. Vallilo, JHEP 0007 (2000) 015, hep-th/0004171.

[4] N. Berkovits, JHEP 0510 (2005) 089, hep-th/0509120.

[5] N. Berkovits and N. Nekrasov JHEP 0612 (2006) 029, hep-th/0609012.

[6] N. Berkovits, JHEP 0503 (2005) 041, hep-th/0406055., JHEP 0909 (2009) 051, hepth/08125074.

[7] N. Berkovits and P. Howe, Nucl. Phys. B635 (2002) 75, hep-th/0112160.

[8] I. Oda and M. Tonin, Phys. Lett. B520 (2001) 398, hep-th/0109051.

[9] O. Chandia and M. Tonin JHEP 0709 (2007) 016, hep-th/07070654.

[10] O. Chandia, JHEP 0607 (2006) 019, hep-th/0604115.

[11] S. Guttenberg, hep-th/08074968.

[12] R. D'Auria, P. Fre', P. A. Grassi and M. Trigiante, JHEP 0807 (2008) 059, hepth/08031703

[13] J. Gomis, D. Sorokin and L. Wulff, JHEP 0908 (2009) 60, [arXiv:0811.1566 [hep-th]]. 
[14] G. Arutyunov and S. Frolov, JHEP 0809 (2008) 129, hep-th/08064940.

[15] B. J. Stefanski, Nucl. Phys. B 808, 8087 (2009) hep-th/08064948 .

[16] R. D'Auria, P. Fre', P. A. Grassi and M. Trigiante, Phys.Rev. D 79, (2009) 086001 hep-th 08081282

[17] G. Bonelli, P. A. Grassi and H. Safaai, JHEP 0810, 085 (2008), hep-th/0808.1051.

[18] R. Stora, Cargese Lectures, Sept.1-15, 1983, Nato ADV.Study.Ser.Phys. bf 115,1 (1984).

[19] L. Bonora and M. Tonin Phys.Lett. B98 (1981) 48

[20] N. Dragon, Z.Phys. C2 (1979) 29

[21] P. S. Howe and P. C. West, Nucl. Phys. B 238, 181 (1984).

[22] J. L. Carr, S. J. J. Gates and R. N. Oerter, Phys. Lett. B 189, 68 (1987).

[23] B. Zumino, Lectures at the Les Houches School Aug 8 - Sept 2, 1983

[24] L. Bonora and P. Cotta-Ramusino,Commun.Math.Phys. 87 (1983) 589

[25] J. Manes, R. Stora and B. Zumino Commun. Math. Phys. 102 (1985),157

[26] D. Anselmi and P. Fre, Nucl. Phys. B 392 (1993) 401 arXiv:hep-th/9208029.

[27] L. Baulieu, M. P. Bellon and R. Grimm, Nucl. Phys. B 294, 279 (1987).

[28] D. P. Sorokin, V. I. Tkach and D. V. Volkov, Mod. Phys. Lett. A 4, 901 (1989), M. Tonin,Phys.Lett. 266 (1991) 312, N. Berkovits, Nucl.Phys. B 350(1991) 193 F. Delduc,A. Galperin, P. Howe,E. Sokatchev, Phys. Rev. D 47 (1993) 578 I. A. Bandos, D. P. Sorokin, M. Tonin, P. Pasti and D. V. Volkov, Nucl. Phys. B 446, 79 (1995) hep-th/9501113, P. S. Howe and E. Sezgin, Phys. Lett. B 390, 133 (1997) hep-th/9607227, D. P. Sorokin, Phys. Rept. 329, 1 (2000) hep-th/9906142.

[29] M. Matone, L. Mazzucato, I. Oda, D. Sorokin and M. Tonin, Nucl. Phys. B 639, 182 (2002), hep-th/0206104.

[30] N. Berkovits, JHEP 0209 (2002) 051, hep-th/ 0201151

[31] P. Fré and P. A. Grassi, Nucl. Phys. B 763 (2007) 1 arXiv:hep-th/0606171.

[32] I. Oda and M. Tonin, Phys. Lett. B 606, 218 (2005), :hep-th/0409052.

[33] I. Oda and M. Tonin, Nucl. Phys. B 727, 176 (2005), hep-th/0505277.

[34] I. Oda and M. Tonin, Nucl. Phys. B 779, 63 (2007), hep-th/07041219

[35] J. Hoogeven and K. Skenderis, JHEP 0711 (2007) 081 hep-th/ 07102598 